\title{
Chemotaxonomic characterization of the thaumarchaeal lipidome
}

Running title: Comparative analysis of the thaumarchaeal lipidome

Felix J. Elling ${ }^{1+}$, Martin Könneke ${ }^{1,2 \#}$, Graeme W. Nicol ${ }^{3}$, Michaela Stieglmeier ${ }^{4}$, Barbara Bayer $^{5}$, Eva Spieck ${ }^{6}$, José R. de la Torre ${ }^{7}$, Kevin W. Becker ${ }^{1 \dagger}$, Michael Thomm ${ }^{8}$, James I. Prosser $^{9}$, Gerhard J. Herndl ${ }^{5,10}$, Christa Schleper ${ }^{4}$, Kai-Uwe Hinrichs ${ }^{1}$

1 Organic Geochemistry Group, MARUM - Center for Marine Environmental Sciences \& Department of Geosciences, University of Bremen, 28359 Bremen, Germany.

${ }^{2}$ Marine Archaea Group, MARUM - Center for Marine Environmental Sciences \& Department of Geosciences, University of Bremen, 28359 Bremen, Germany.

3 Environmental Microbial Genomics, Laboratoire Ampère, École Centrale de Lyon, Université de Lyon, 69134 Ecully, France

${ }^{4}$ Department of Ecogenomics and Systems Biology, Center of Ecology, University of Vienna, 1090 Vienna, Austria.

${ }^{5}$ Department of Limnology and Bio-Oceanography, Center of Ecology, University of Vienna, 1090 Vienna, Austria.

${ }^{6}$ Biocenter Klein Flottbek, Department of Microbiology and Biotechnology, University of Hamburg, 22609 Hamburg, Germany.

${ }^{7}$ Department of Biology, San Francisco State University, San Francisco, CA, USA.

8 Lehrstuhl für Mikrobiologie und Archaeenzentrum, Universität Regensburg, 93053 Regensburg, Germany.

${ }^{9}$ Institute of Biological and Environmental Sciences, University of Aberdeen, Cruickshank Building, Aberdeen, AB24 3UU, United Kingdom.

${ }^{10}$ Department of Marine Microbiology and Biogeochemistry, Royal Netherlands Institute for Sea Research, Utrecht University, 1790 AB Den Burg, Texel, The Netherlands

\#Corresponding author. Tel.: + 49421218 65747. Fax: +49 421218 65715. E-mail: mkoenneke@marum.de

+present address: Department of Earth and Planetary Sciences, Harvard University, Cambridge, MA 02138, USA.

${ }^{\dagger}$ present address: Department of Marine Chemistry and Geochemistry, Woods Hole Oceanographic Institution, Woods Hole, MA 02543, USA.

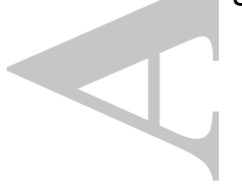

This article has been accepted for publication and undergone full peer review but has not been through the copyediting, typesetting, pagination and proofreading process which may lead to differences between this version and the Version of Record. Please cite this article as an 'Accepted Article', doi: 10.1111/1462-2920.13759 


\section{Originality-Significance Statement}

Archaeal lipids are frequently used as biomarkers in biogeochemistry and microbial ecology, with applications ranging from chemotaxonomic characterization and stable isotope probing of uncultured and 'unculturable' microbial communities to the reconstruction of climatic conditions from ancient sediments. Interpretation of these lipid profiles relies on detailed knowledge of lipid composition and membrane adjustment mechanisms in cultivated archaea. However, the detailed intact polar lipid compositions of widely distributed Thaumarchaeota are yet not well characterized. Here we describe in detail the lipidomes of ten established thaumarchaeal cultures from soils, hydrothermal springs, and the ocean in order to uncover the chemotaxonomic potential of thaumarchaeal lipids as specific biomarkers and potential adaptation strategies employed by this environmentally relevant archaeal phylum.

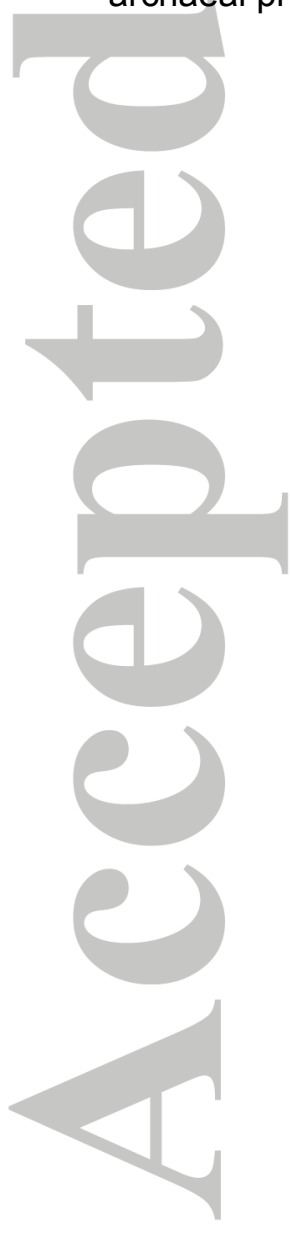




\section{Summary}

Thaumarchaeota are globally distributed and abundant microorganisms occurring in diverse habitats and thus represent a major source of archaeal lipids. The scope of lipids as taxonomic markers in microbial ecological studies is limited by the scarcity of comparative data on the membrane lipid composition of cultivated representatives, including the phylum Thaumarchaeota. Here, we comprehensively describe the core and intact polar lipid (IPL) inventory of ten ammonia-oxidizing thaumarchaeal cultures representing all four characterized phylogenetic clades. IPLs of these thaumarchaeal strains are generally similar and consist of membrane-spanning, glycerol dibiphytanyl glycerol tetraethers with monoglycosyl, diglycosyl, phosphohexose and hexose-phosphohexose headgroups. However, the relative abundances of these IPLs and their core lipid compositions differ systematically between the phylogenetic subgroups, indicating high potential for chemotaxonomic distinction of thaumarchaeal clades. Comparative lipidomic analyses of 19 euryarchaeal and crenarchaeal strains suggested that the lipid methoxy archaeol is synthesized exclusively by Thaumarchaeota and may thus represent a diagnostic lipid biomarker for this phylum. The unprecedented diversity of the thaumarchaeal lipidome with 118 different lipids suggests that membrane lipid composition and adaptation mechanisms in Thaumarchaeota are more complex than previously thought and include unique lipids with as yet unresolved properties.

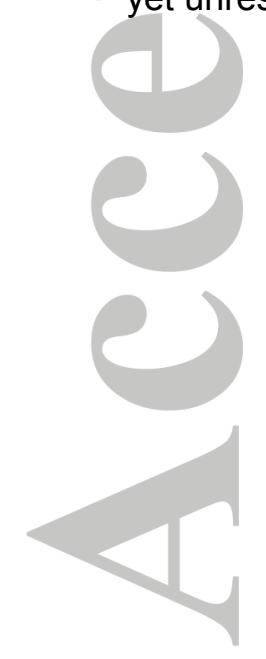




\section{Introduction}

Archaea of the phylum Thaumarchaeota are globally distributed microorganisms accounting for up to $20 \%$ of the picoplankton in the oceans (Karner et al., 2001; Schattenhofer et al., 2009) and $1-5 \%$ of the prokaryotes in soil (Ochsenreiter et al., 2003; Brochier-Armanet et al., 2008; Lehtovirta et al., 2009; Stahl and de la Torre, 2012). Following the isolation of the first representative Ca. Nitrosopumilus maritimus (Könneke et al., 2005), Thaumarchaeota have become recognized as major contributors to ammonia oxidation in a wide range of habitats including the marine water column and sediment as well as terrestrial, limnic, and geothermal systems (Francis et al., 2005; Leininger et al., 2006; Auguet and Casamayor, 2008; de la Torre et al., 2008; Hatzenpichler et al., 2008; Prosser and Nicol, 2008; Reigstad et al., 2008; Dodsworth et al., 2011; Lehtovirta-Morley et al., 2011). All characterized Thaumarchaeota are chemolithoautotrophs generating energy by the oxidation of ammonia to nitrite (Stahl and de la Torre, 2012) and fixing $\mathrm{CO}_{2}$ via a hydroxypropionate/hydroxybutyrate cycle (Walker et al., 2010; Könneke et al., 2014). The phylum Thaumarchaeota is commonly subdivided into several subgroups based on ammonia monooxygenase subunit $\mathrm{A}(\mathrm{amo} A)$ and $16 \mathrm{~S}$ rRNA gene phylogenies that broadly correlate with habitat types (Fig. 1; Brochier-Armanet et al., 2008; Spang et al., 2010; Pester et al., 2011; Stahl and de la Torre, 2012). Ca. N. maritimus as well as most marine thaumarchaeal sequences, and to a lesser extent soil and lacustrine sequences, are affiliated with Group 1.1a (Fig. 1; Francis et al., 2005; Könneke et al., 2005; Pester et al., 2012; Stahl and de la Torre, 2012). The SAGMCG-1/Nitrosotalea cluster represents a sister group of the Group 1.1a Thaumarchaeota comprising environmental sequences from soils and lakes as well as two acidophilic isolates from soil, Ca. Nitrosotalea devanaterra and Ca. Nitrosotalea sp. strain Nd2 (Fig. 1; Lehtovirta-Morley et al., 2011, 2014; Stahl and de la Torre, 2012; Auguet and Casamayor, 2013). While Group 1.1a Thaumarchaeota are also found in soils (e.g., Pester et al., 2011), most sequences from soils and other terrestrial environments as well as the isolate Nitrososphaera viennensis (Tourna et al., 2011; Stieglmeier et al., 2014) are affiliated with Group 1.1b (Fig. 1; Bintrim et al., 1997; DeLong, 1998; Stahl and de la Torre, 2012). Additionally, Group 1.1a and 1.1b both 
contain moderate thermophiles such as $\mathrm{Ca}$. Nitrosotenuis uzonensis and $\mathrm{Ca}$. Nitrososphaera gargensis, which grow in a temperature range of $28-52{ }^{\circ} \mathrm{C}$ and $35-46{ }^{\circ} \mathrm{C}$, respectively (Hatzenpichler et al., 2008; Lebedeva et al., 2013). However, the only cultivated obligate thermophile is Ca. Nitrosocaldus yellowstonii (Fig. 1; ThAOA/HWCG-III cluster), which was enriched from a Yellowstone hot spring and grows in a temperature range of $60{ }^{\circ} \mathrm{C}$ to $74{ }^{\circ} \mathrm{C}$ (de la Torre et al., 2008). Furthermore, cultivation-independent surveys indicate that several additional lineages of Thaumarchaeota occur in the environment for which no cultivated representative and limited observational data exist (Schleper et al., 2005; Nicol and Schleper, 2006; Stahl and de la Torre, 2012).

Detection of Thaumarchaeota in the environment is commonly achieved by PCR-based marker gene surveys or metagenomic approaches (Ochsenreiter et al., 2003; Francis et al., 2005) and the analysis of characteristic glycerol dibiphytanyl glycerol tetraether (GDGT, Fig. 2) membrane lipids (e.g., Leininger et al., 2006; Coolen et al., 2007; Wakeham et al., 2007; Schouten et al., 2012). While providing lower taxonomic resolution than molecular biological techniques, lipid analysis offers PCR-independent, qualitative and quantitative analysis of major clades of Archaea and Bacteria (Sturt et al., 2004). Additionally, carbon isotopic analysis of microbial lipids enables insights into predominant metabolisms and activity of microorganisms (Hinrichs et al., 1999; Pearson et al., 2001; Biddle et al., 2006; Schubotz et al., 2011). GDGTs from planktonic Thaumarchaeota accumulate in sediments and are broadly used by geochemists for reconstructing past sea surface temperatures using the $\mathrm{TEX}_{86}$ paleothermometer, which is based on temperature-dependent variations in GDGT alkyl-chain cyclization (Schouten et al., 2002). Application of these lipid-based approaches to complex environmental samples relies on detailed knowledge of the phylogenetic distribution of characteristic marker lipids as well as functional and ecological constraints. However, only a limited set of lipids, consisting mainly of monoglycosidic, diglycosidic and glycophosphatidic GDGTs was reported from cultivated marine and terrestrial Thaumarchaeota (Schouten et al., 2008; Pitcher et al., 2011; Sinninghe Damsté et al., 2012). 
Hitherto, relative abundances of intact polar lipid (IPL) classes as well as their corresponding core lipid compositions have been examined in only few Thaumarchaeota. The mesophilic marine pure culture Ca. N. maritimus and two related strains have been studied in detail (Elling et al., 2014, 2015) by recently developed analytical methods that allow the simultaneous quantification of relative abundances of individual IPL classes as well as their core GDGT composition (Zhu et al., 2013). For instance, the Ca. N. maritimus lipidome analyzed with these methods revealed higher lipid diversity than previously recognized for any thaumarchaeon, including major abundances of diether lipids as well as a novel putative biomarker for Thaumarchaeota, methoxy archaeol (Elling et al., 2014, 2015). Application of these methods to recently cultivated thaumarchaeal cultures from a broad range of habitats will enable the screening for novel lipid biomarkers. Furthermore, the characterization of the lipid inventory in cultivated Thaumarchaeota will facilitate the interpretation of IPLs abundantly detected in environmental samples and their assignment to potential source organisms.

\section{Results}

In this study, we dissected the lipidome of cultivated Thaumarchaeota representing the four main phylogenetic subgroups and originating from soils, hydrothermal springs and the ocean's surface water. Thaumarchaeal pure or enrichment cultures were grown in multiple laboratories as batch cultures and harvested in late exponential or early stationary phase. Using state-of-the-art ultra-high performance liquid chromatography (UPLC) connected to ultra-high resolution quadrupole time-of-flight tandem mass spectrometer (MS), the lipid inventories of seven previously analyzed strains were significantly extended and the lipid compositions of three thaumarchaeal strains were analyzed for the first time (Fig. 3). Relative abundances of core and intact polar lipids are tabulated in Table 1 and S1 as well as in the supplementary data file. Hierarchical cluster analysis was performed separately on the core and intact polar lipid abundances to investigate the relationships between the lipidomes of the ten thaumarchaeal strains (Fig. 4). Simpson diversity indices were calculated based on 
full lipid diversity including individual cyclized core and intact polar GDGTs, respectively (Table 1, Fig. S2). The influence of conservative growth parameters (temperature, $\mathrm{pH}$, salinity) on lipid composition were investigated by multivariate statistics, including redundancy analysis (RDA, assuming a linear model), constrained correspondence analysis (CCA, assuming a unimodal model with potential to capture bimodal distributions; Ramette, 2007), and non-metric multidimensional scaling (NMDS). Results of statistical analyses are described separately below for core/apolar and intact polar lipids.

\section{Common patterns in the lipidomes of cultivated Thaumarchaeota}

A total of 118 individual lipids, representing either core lipids or IPLs or quinones, were identified in the ten analyzed thaumarchaeal cultures (Fig. 3, S1). Forty lipid compounds were common to all thaumarchaeal strains, while 11 compounds were unique to Group $1.1 \mathrm{~b}$ Thaumarchaeota, 27 compounds were found only in Group 1.1a Thaumarchaeota and there were no unique compounds in the SAGMCG-1 and HWCG-III groups (Fig. 3). The most complex and diverse lipid inventory was found within the Group 1.1a Thaumarchaeota, represented by five marine isolates of the genus Nitrosopumilus, with a total number of 86 distinct compounds. This diversity does not represent an artifact from the higher number of analyzed Group 1.1a Thaumarchaeota compared to the other clades, as all Group 1.1a strains produce the same lipid types. The thaumarchaeal lipidome comprises as core lipids, among others, acyclic and cyclized GDGTs, glycerol dialkanol diethers (GDDs) and archaeols. Common lipid headgroups were monoglycosyl (1G), diglycosyl (2G), hexosephosphohexose $(\mathrm{HPH})$, and phosphohexose $(\mathrm{PH})$ and the affiliation of these headgroups with cyclized GDGTs varied systematically between strains (Fig. 5).

\section{Core and apolar lipids}

Analysis of the core lipid fractions derived from hydrolysis of total lipids revealed distinct distributions of glycerol diphytanyl diethers (archaeols, AR), GDGTs, hydroxylated GDGTs (OH-GDGTs), and glycerol trialkyl glycerol tetraethers (GTGTs, for structures refer to Fig. 2; 
De Rosa et al., 1983) among the investigated cultures. Major core lipid types found in all thaumarchaeal strains were GDGTs with zero to four cyclopentane moieties (GDGT-0 to GDGT-4), crenarchaeol (a GDGT containing four cyclopentane moieties and one cyclohexane moiety) and methoxy archaeol (MeO-AR). Up to four isomers of each GDGT, with so far unresolved stereochemistry, were eluted before and after the typical GDGT peaks (Pitcher et al., 2011; Sinninghe Damsté et al., 2012; Becker et al., 2013; Elling et al., 2014). The relative abundances of these isomers varied systematically between the thaumarchaeal clades (Fig. 6), e.g., the GDGT-2a isomer was more abundant than GDGT-2 in Group 1.1b while GDGT-2 was dominant in Group 1.1a Thaumarchaeota (Fig. 6c). MeO-GDGTs were detected as trace components $(<0.1 \%)$ in all strains. Acyclic GTGT (GTGT-0) and monounsaturated GTGT-0 (GTGT-0:1; Elling et al., 2014) were detected in all thaumarchaeal strains. A GTGT with one cyclopentane moiety was detected in N. viennensis strains EN76 and EN123. GTGTs with 1-4 cyclopentane moieties were detected in Ca. N. gargensis and Ca. N. yellowstonii. The ring-containing GTGTs could be distinguished from unsaturated GTGTs by their elution order in reversed phase UPLC, i.e., ring-containing GTGTs eluted after the acyclic saturated GTGT while unsaturated GTGTs eluted prior to the acyclic saturated GTGT, analogously to unsaturated and ring-containing GDGTs (cf. Zhu et al., 2013). Ring indices and TEX $_{86}$ calculated from total GDGTs (excluding isomers other than the crenarchaeol regioisomer) differed significantly between the cultures (Table 1); both variables were linearly correlated with growth temperature across the different strains when data from Ca. N. yellowstonii was excluded (Fig. S2). Group 1.1b Thaumarchaeota showed the highest ring indices (4.3-4.8) and TEX ${ }_{86}$ values (0.97-0.99). The lowest ring index and TEX $_{86}$ values were observed in strain NAOA6 (2.7) and Ca. Nitrosocaldus yellowstonii, respectively (0.61).

Among the forty shared compounds of the thaumarchaeal lipidome, MeO-AR was identified as one of the most abundant lipid compounds, accounting for up to $20 \%$ in the acidophilic thaumarchaeon $\mathrm{Ca}$. N. devanaterra and for $2-11 \%$ in the marine strains (Table 1). Comparative analysis of 19 cultured representatives of the phyla Crenarchaeota and 
Euryarchaeota, with lipids extracted and analyzed using the same protocols, revealed that MeO-AR, like crenarchaeol, was synthesized exclusively by members of the phylum Thaumarchaeota (Table 2). In contrast, GDGT and GTGT biosynthesis was a common, but not universal, trait among the three archaeal phyla (Table 2).

Cluster analysis indicated that the distribution of core lipid types among Thaumarchaeota is dependent on phylogeny (Fig. 4). The core lipid compositions of all Group 1.1a Thaumarchaeota were closely related to each other with relatively similar distributions of core GDGTs, low crenarchaeol regioisomer contents and the occurrence of OH-GDGTs; the distributions of these compounds were distinct from the other thaumarchaeal lineages. Similarly, MeO-AR contents were higher in Group 1.1a than in most other Thaumarchaeota. The low abundance of OH-GDGT core lipids in contrast to the high abundances of IPLs with OH-GDGT core structures is likely related to the loss of the hydroxyl group during acid hydrolysis (Liu et al., 2012b; Sinninghe Damsté et al., 2012).

The core lipid composition of the soil thaumarchaeon Ca. N. devanaterra was very similar to that of Group 1.1a strains and thus reflected the phylogenetic position of this thaumarchaeon in a sister clade of Group 1.1a, SAGMCG-1. However, Ca. N. devanaterra was distinct from 1.1a Thaumarchaeota by exhibiting higher abundances of GDGT-4 and MeO-AR. In contrast to Group 1.1a cultures, the lipidomes of Group 1.1b Thaumarchaeota were highly divergent. The two investigated Group 1.1b Thaumarchaeota from soil, N. viennensis strains EN76 and EN123, were characterized by high abundances of GDGT-4, the crenarchaeol regioisomer, and GDDs. In contrast, the lipidome of the moderately thermophilic Group $1.1 \mathrm{~b}$ thaumarchaeon Ca. N. gargensis was nearly completely composed of crenarchaeol and its regioisomer. The thermophilic Thaumarchaeota of the HWCG-III cluster were distinct from the other thaumarchaeal clades due to GTGTs being their dominant core lipids as well as relatively high amounts of crenarchaeol compared to the other GDGTs.

CCA and RDA indicated temperature and salinity as major factors driving core lipid composition (Fig. S4a, Table S2-S5), with RDA yielding lower explanatory power, potentially 
indicating non-linearity. In particular, high salinity was associated with high relative abundances of GDGT-0 through GDGT-2 and OH-GDGTs while high temperature was associated with GTGTs. The strong correlation of core lipid composition with salinity is not observed in single Group 1.1a species grown at different salinities (Elling et al., 2015) and might thus reflect a phylogenetic or physiological divide between Group 1.1a and other Thaumarchaeota.

\section{Intact polar lipids (IPLs) and respiratory quinones}

Reversed phase UPLC-MS analyses revealed a high diversity of IPLs among the investigated thaumarchaeal strains (Fig. 4b, S1). The major IPLs in all strains were GDGTs with monoglycosyl (1G), diglycosyl (2G), phosphohexose $(\mathrm{PH})$ and hexose-phosphohexose $(\mathrm{HPH})$ headgroups. However, small amounts of intact polar archaeols with $1 \mathrm{G}$ and $\mathrm{PH}$ headgroups were detected in all thaumarchaeal strains, while no intact polar GTGTs were observed. 2G-GDGTs and 2G-OH-GDGTs each consisted of two series of isomers that were separated chromatographically but yielded similar $\mathrm{MS}^{2}$ fragmentation spectra (Elling et al., 2014; Fig. S1). Intact polar GDGTs containing modified glycosidic headgroups, such as methylated- (1MeG-1G- and 1MeG-2G-GDGTs) and deoxy-sugars (1deoxyG-GDGTs), were detected as trace components in some Thaumarchaeota (Fig. 3). In addition, minor amounts of $\mathrm{OH}-G D D s$ were detected in the total lipid extracts of all investigated Group 1.1a Thaumarchaeota as well as $\mathrm{Ca}$. N. devanaterra, but were not detected in the hydrolyzed lipid extracts. Two isoprenoid naphthoquinones were detected in all thaumarchaeal strains and were identified as menaquinones with fully unsaturated $\left(\mathrm{MK}_{6: 0}\right)$ and monounsaturated $\left(\mathrm{MK}_{6: 1}\right)$ side chains composed of six isoprenoid units (Elling et al., 2016). While the relative abundances of these quinones varied between different strains, $\mathrm{MK}_{6: 0}$ was more abundant than $\mathrm{MK}_{6: 1}$ in all thaumarchaeal strains (Table S1).

In contrast to the core lipids, cluster analysis of the IPLs indicated that the thaumarchaeal strains could be divided into three major groups related to habitat type/growth conditions (Fig. 4): terrestrial thermophiles, marine mesophiles, and soil mesophiles. The terrestrial 
thermophiles were characterized by relatively high abundances of $1 \mathrm{G}-\mathrm{GDGTs}$ and HPHGDGTs as well as intact polar archaeols. All marine mesophiles shared high abundances of 2G-GDGTs and 2G-OH-GDGTs compared to the other Thaumarchaeota, while HPH-GDGTs were abundant only in some strains. The soil Thaumarchaeota ( $N$. viennensis EN76 and EN123, Ca. N. devanaterra) were characterized by high relative abundances of 1G-GDGTs and 2G-GDGTs as well as 1G-GDDs in N. viennensis. While these groupings were in part related to phylogeny (e.g., all investigated marine mesophiles are Group 1.1a Thaumarchaeota), the distinct clustering suggests that IPL composition in Thaumarchaeota may reflect habitat type, i.e., combined effects of a set of environmental constraints. However, neither RDA, CCA, or NMDS revealed significant correlations of relative abundances of major IPL groups or individual IPLs with temperature, $\mathrm{pH}$, or salinity. This implies that IPL modification patterns are not consistent across major clades and may only be assessed in distinct sub-clades or species.

\section{Discussion}

Chemotaxonomic characteristics of the thaumarchaeal lipidome

We discovered an unprecedented diversity of membrane lipids in the phylum Thaumarchaeota and extended the thaumarchaeal lipidome by characterizing 118 structurally different lipids. Despite the overall similarity in the types of lipids synthesized by the major thaumarchaeal clades, membrane lipid composition varies widely among phylogenetic clades and thus our study supports the potential of thaumarchaeal membrane lipids as chemotaxonomic markers and their application in microbial ecology. The interpretation of the lipid profiles reported here is limited by the fact that replicate cultures were available only for some strains ( $N$. maritimus SCM1, NAOA2, NAOA6). However, duplicate cultivation and our previous studies show that lipid relative abundances and TEX are reproducible (Elling et al., 2014, 2015). The variability between batches, including all biological and analytical artifacts, is lower $(<10 \%$, Fig. S3) than the variability observed between major clades of Thaumarchaeota and among most Group 1.1a strains (Fig. 4). The 
compositions of growth media were kept as similar as possible, with all Group 1.1a Thaumarchaeota grown on the same medium and the other strains grown on media derived from SCM, with only small variations owing to $\mathrm{pH}$, salinity and specific growth requirements (e.g., supplementation of pyruvate to Nitrososphaera spp. media). Importantly, the chemical species of essential nutrients, such as phosphorous (as $\mathrm{KH}_{2} \mathrm{PO}_{4}$ ), nitrogen (as $\mathrm{NH}_{4} \mathrm{Cl}$ ), sulfur (as $\mathrm{SO}_{4}{ }^{2-}$ ), and inorganic carbon (as $\mathrm{NaHCO}_{3}$ ) were identical and provided in a similar concentration range. In contrast, changes in environmental parameters and growth state (Elling et al., 2014, 2015; Qin et al., 2015; Hurley et al., 2016) could significantly blur differences in lipid abundances between thaumarchaeal clades. Additionally, the effects of low levels of bacterial contaminants in the studied enrichment cultures are not known. Considering these limitations, we emphasize that the lipid profiles shown here reflect the capacities of each strain under optimal laboratory conditions.

Comparison of the ten investigated lipidomes indicates that the core lipid compositions of Thaumarchaeota reflect their affiliation with phylogenetic subgroups (Fig. 4a). In contrast, IPLs appear to reflect the habitat type or growth conditions (Fig. 4b), but this assessment hinges on the placement of just two strains, Ca. N. gargensis and Ca. N. devanaterra, while it is not driven by the large number of marine Group 1.1a Thaumarchaeota, which all originate from a marine habitat. Future analysis of Group 1.1a Thaumarchaeota from soil (e.g., Ca. Nitrosoarchaeum koreensis; Jung et al., 2011) and hot springs (Ca. N. uzonensis; Lebedeva et al., 2013) may allow evaluation of whether thaumarchaeal IPLs may be used as habitatspecific biomarkers.

In most thaumarchaeal strains investigated here, 1G-GDGTs are the most abundant IPLs. Similarly, 1G-GDGTs, in particular 1G-crenarchaeol, are often the most abundant archaeal lipids detected in the marine water column (Schubotz et al., 2009; Schouten et al., 2012; Wakeham et al., 2012; Basse et al., 2014; Xie et al., 2014). However, 1G-GDGTs have been suggested to be more refractory than 2G- and HPH-GDGTs when released into the environment after cell lysis, and may therefore be less suitable for tracing living biomass than 
other thaumarchaeal polar lipids (e.g., Schouten et al., 2012). Relatively high abundances of HPH-GDGTs during growth of $\mathrm{Ca}$. N. maritimus compared to the stationary phase suggest that HPH-GDGTs might be indicators of metabolically active Thaumarchaeota (Elling et al., 2014). Examination of the five marine planktonic thaumarchaeal strains in our dataset indicates that HPH-GDGT abundances are highly variable in Group 1.1a Thaumarchaeota (Fig. 4), i.e., the dominant thaumarchaeal clade in the ocean. Therefore, changes in HPHGDGT abundances in the marine water column might not only be related to thaumarchaeal abundances and metabolic activity but may also reflect changes in thaumarchaeal community composition. Thus, $2 \mathrm{G}-\mathrm{GDGTs}$ and $2 \mathrm{G}-\mathrm{OH}-\mathrm{GDGT}$ seem to be most suitable for tracing planktonic thaumarchaeal biomass due to their high relative abundances in all strains. OH-GDGTs and their intact polar derivatives appear to be exclusively synthesized by Group 1.1a (Fig. 3 and 4), consistent with previous investigations in soil and sedimentary thaumarchaeal cultures (Pitcher et al., 2011; Sinninghe Damsté et al., 2012), and appear to be well suited for tracing this group in aquatic settings. Complimentary analysis of the thermophile $\mathrm{Ca}$. N. uzonensis could reveal whether OH-GDGTs are specific for mesophilic Group 1.1a Thaumarchaeota or if they are a common feature of this thaumarchaeal clade.

Thaumarchaeal communities in soil are commonly dominated by Group $1.1 \mathrm{~b}$ Thaumarchaeota related to N. viennensis (e.g., Pester et al., 2012), but the distribution of Thaumarchaeota related to Ca. N. devanaterra (SAGMCG-1 cluster) may largely be restricted to acidic soils (e.g., Gubry-Rangin et al., 2011). Both clades may be distinguishable in environmental samples by their distinct lipid compositions. While both groups synthesize predominantly 1G-GDGTs with GDGT-4 and crenarchaeol as core lipids, Ca. N. devanaterra synthesizes 2G-GDGTs predominantly with GDGT-3 as the core lipid, in contrast to 2GGDGT with GDGT-4 in N. viennensis (Fig. 5). Furthermore, N. viennensis contains high abundances of 1G- and 2G-derivatives of the crenarchaeol regioisomer as well as 1G-GDDs, while Ca. N. devanaterra contains high proportions of MeO-AR (Fig. 4, Table 1). Additionally, small amounts of 3G-GDGTs are found only in Group 1.1b Thaumarchaeota. 
Thermophilic Thaumarchaeota associated with the HWCG-III cluster and Group 1.1b have overall similar IPL profiles but are distinguished by the high abundance of the crenarchaeol regioisomer in Group 1.1b Thaumarchaeota and high abundances of acyclic and ringcontaining GTGTs in HWCG-III Thaumarchaeota. The high abundances of GTGTs in HWCG-III Thaumarchaeota are unprecedented among cultivated archaea (Table 2; cf. De Rosa and Gambacorta, 1988; Knappy et al., 2011, 2015; Feyhl-Buska et al., 2016) and have previously been interpreted to reflect sub-optimal growth conditions of $\mathrm{Ca}$. N. yellowstonii (cf. de la Torre et al., 2008). However, growth conditions for Ca. $\mathrm{N}$ yellowstonii were optimal in our experiment and cells were harvested during late growth phase (Fig. S5). The role of GTGTs remains unresolved but their high abundance might represent a unique characteristic of this thermophilic thaumarchaeal clade. Alternatively, GTGTs could represent intermediates or side products of GDGT biosynthesis formed by incomplete side chain linkage of two archaeol moieties (cf. Pearson, 2014). Furthermore, Ca. N. gargensis (Group 1.1b) synthesizes trace amounts of intact polar GDGTs with a combination of methylated and nonmethylated glycosidic headgroups (1MeG-1G- and 1MeG-2G-GDGTs; cf. Pitcher et al., 2010), which were not detected in any other characterized thaumarchaeal strain.

High abundances of core and 1G-GDDs are characteristic of both $N$. viennensis strains and distinguish them from other Thaumarchaeota. GDD core lipids were initially detected in marine sediments (Knappy and Keely, 2012; Liu et al., 2012a, 2012c) and in soils (Yang et al., 2014; Coffinet et al., 2015). Because of their chemical structure, GDDs have been discussed to represent either intermediates of GDGT biosynthesis (Liu et al., 2012a; Meador et al., 2014b; Villanueva et al., 2014) or degradation products of GDGTs (Knappy and Keely, 2012; Liu et al., 2012a, 2016; Yang et al., 2014). GDDs were also reported in several methanogens (Liu et al., 2012a; Bauersachs et al., 2015) and as 1G-GDDs in Ca. N. maritimus (Elling et al., 2014; Meador et al., 2014b). The abundance of 1G-GDDs in various thaumarchaeal cultures indicates that GDDs may be regular membrane components and that IPL-GDDs detected in marine sediments and soils may thus originate from living Thaumarchaeota. 
Diagnostic lipid biomarkers for Thaumarchaeota

Comparison of the thaumarchaeal lipidome with the lipid inventory of 19 crenarchaeal and euryarchaeal species revealed that Thaumarchaeota harbor distinct biomarkers as well as lipids common to all archaea.

Regarding unique lipid compounds with potential to serve as biomarker for the phylum Thaumarchaeota, our study confirmed the presence of crenarchaeol, a well-established lipid biomarker initially reported to occur in pelagic archaea (Sinninghe Damsté et al., 2002, 2012;

Pitcher et al., 2011), in all thaumarchaeal but not in any euryarchaeal or crenarchaeal species investigated (Table 2). It has previously been suggested that crenarchaeol and other GDGTs are also produced by uncultured Marine Group 2 Euryarchaeota based on the covariation of 16S rRNA gene and GDGT abundances at Hawaii Ocean Time-series Station ALOHA (Lincoln et al., 2014a). However, the relative contributions of Marine Group 1 Thaumarchaeota and Marine Group 2 Euryarchaeota to the marine GDGT pool remain disputed (Lincoln et al., 2014b; Schouten et al., 2014). All analyzed thaumarchaeal strains synthesized the same suite of respiratory quinones, the menaquinones $\mathrm{MK}_{6: 0}$ and $\mathrm{MK}_{6: 1}$ (Table 2, S1). While $\mathrm{MK}_{6: 0}$ and $\mathrm{MK}_{6: 1}$ are minor quinones in some thermophilic Crenarchaeota (e.g., Desulfurococcales) and Euryarchaeota (e.g., Archaeoglobales), they have not been detected in other cultivated mesophilic archaea (Elling et al., 2016). Therefore, these menaquinones, especially when dominating quinone distributions, may be regarded as biomarkers for the phylum Thaumarchaeota in low-temperature marine and terrestrial habitats.

The apolar lipid MeO-AR (Elling et al., 2014) was present in all investigated thaumarchaeal strains but not in any analyzed crenarchaeal or euryarchaeal species (Table 2). Hence, MeO-AR has a high potential as a diagnostic biomarker for members of the phylum Thaumarchaeota. Because of its relatively high abundance in Group 1.1a Thaumarchaeota (Table 1), MeO-AR could be used for determining the contribution of Thaumarchaeota to total archaeal biomass, e.g., by relating the abundance of MeO-AR to that of archaeol, a 
universal archaeal lipid biomarker (Table 2). In contrast to the core GDGT crenarchaeol, MeO-AR represents a major thaumarchaeal lipid that can be analyzed by gas chromatography, e.g., for isotopic analysis without the need of previous ether cleavage or derivatization.

In addition to lipids specific for the phylum Thaumarchaeota, we found at least minor amounts of archaeol in all investigated archaea (Fig. 4). This finding contrasts with those of prior studies that did not report the occurrence of archaeol in most cultivated Thaumarchaeota, including some of the same strain studied here (Pitcher et al., 2011; Sinninghe Damsté et al., 2012; Villanueva et al., 2014; Lehtovirta-Morley et al., 2016). Instead, our results, combined with previously published data (Shimada et al., 2002; Koga and Morii, 2005; Tarui et al., 2007), indicate that archaeol may be regarded as a universal lipid of the domain Archaea.

Implications for the TEX 86 paleotemperature proxy

Fossil core GDGTs of marine planktonic Thaumarchaeota preserved in sediments are valuable biomarkers for reconstructing ancient ocean temperatures using the TEX $\mathrm{X}_{86}$ index. This proxy is based on the fact that many thermophilic archaea, and by extension also mesophilic Thaumarchaeota, regulate GDGT cyclization in response to temperature (De Rosa et al., 1980; Schouten et al., 2002; Oger and Cario, 2013, and references therein). The specific ratio indexed in $\mathrm{TEX}_{86}$, i.e., the relative abundances of GDGT-2 and GDGT-3 versus GDGT-1 through GDGT-3 and the crenarchaeol regioisomer, found in core-top sediments yielded the best correlation with sea surface temperature (Schouten et al., 2002). In the strains studied here, the weak linear correlation between $\mathrm{TEX}_{86}$ and temperature contrasted with the more robust relationship between GDGT cyclization and temperature (Table 1, Fig. S2), suggesting that the $\mathrm{TEX}_{86}$ ratio does not uniformly reflect membrane response to temperature across a diverse group of marine and non-marine Thaumarchaeota. Similarly, previous studies have shown that $\mathrm{TEX}_{86}$-temperature relationships (slopes and offsets) differ even among closely related Group 1.1a Thaumarchaeota (Elling et al., 2015; Qin et al., 2015; 
Zhu et al., 2016). Taken together, these observations indicate that the deviations and scatter in the environmental $\mathrm{TEX}_{86}$ signal and global calibration could be driven both by deviations from optimum growth conditions and differences in community composition.

Moreover, in the marine and terrestrial mesophilic Thaumarchaeota studied here, each intact polar GDGT class is preferentially associated with specific core GDGTs (Fig. 5). Differences in degradation rates of intact polar GDGTs (e.g., phosphatidic versus glycosidic; Logemann et al., 2011; Schouten et al., 2012; Xie et al., 2013) may influence the release rates of core GDGTs from their polar precursors and thus impact the $\mathrm{TEX}_{86}$ index (cf. Elling et al., 2014). Although degradation rates of intact polar GDGTs remain unknown, ocean or lake surface temperatures may be significantly overestimated in environments with high terrigenous input, either due to the higher relative abundance of the crenarchaeol regioisomer in soil Group 1.1b versus marine Group 1.1a Thaumarchaeota (Fig. 4a), as previously demonstrated for the Yellow river (Wu et al., 2014), or due to the higher ratio of GDGT-3 over GDGT-2 in Group 1.1b (Fig. 5). Terrigenous input of GDGTs could be identified based on the profound differences in the distribution of GDGT isomers among the major thaumarchaeal clades (Fig. 6 ) and its effect on $\mathrm{TEX}_{86}$ could be reduced by employing advanced chromatographic methods that allow separate quantification of GDGT isomers (e.g., Becker et al., 2013; Hopmans et al., 2016), which co-elute using standard methods.

\section{Patterns in thaumarchaeal membrane lipid adaptation}

Thaumarchaeota inhabit a broad range of habitats covering large gradients of temperature ( -2-97 ${ }^{\circ} \mathrm{C}$; DeLong et al., 1994; Murray et al., 1998; Reigstad et al., 2008), salinity (freshwater to hypersaline; Auguet et al., 2010; Ngugi et al., 2015), and pH ( 2-8; Nicol et al., 2008; Reigstad et al., 2008; Lehtovirta-Morley et al., 2011) and thus must employ a variety of mechanisms for regulating optimal membrane functionality.

In contrast to the prevalence of bilayer-forming archaeols in other mesophilic archaea (Koga and Morii, 2005), all thaumarchaeal species studied here contain membrane-spanning 
GDGTs as their dominant lipids (Table 1, S1). These isoprenoid lipids form monolayermembranes with highly reduced water and proton permeability compared to (non-isoprenoid) bilayer membranes (Yamauchi et al., 1993; van de Vossenberg et al., 1998; Mathai et al., 2001; Konings et al., 2002), thus maximizing the maintenance of proton motive force and facilitating pH homeostasis (van de Vossenberg et al., 1998; Van de Vossenberg et al., 1998; Baker-Austin and Dopson, 2007; Boyd et al., 2011). Cycloalkylation of GDGTs further reduces membrane fluidity and proton permeability by enabling higher packing densities (Chong, 2010, and references therein).

The high degree of GDGT cycloalkylation (ring index) observed in Thaumarchaeota even at mesophilic growth temperatures (Table 1) may reflect a physiological response to reduce the requirement of reducing equivalents from reverse electron flow at low respiration rates (Hurley et al. 2016). In addition, membranes with a high proportion of cyclic GDGTs offer a high efficiency to maintain the chemiosmotic potential, owing to the low energetic yield of ammonia oxidation (Valentine, 2007).

Similar to thermophilic Euryarchaeota and Crenarchaeota (De Rosa et al., 1980; Oger \& Cario 2013, and references therein), Thaumarchaeota acclimatize to high temperatures by further increasing GDGT cycloalkylation (Elling et al., 2015). Accordingly, the thermophiles Ca. N. yellowstonii and Ca. N. gargensis contain the highest abundances of crenarchaeol relative to other GDGTs (Table S1). However, the highest ring indices were not observed in Ca. N. yellowstonii, the thaumarchaeal culture grown at the highest temperatures $\left(72{ }^{\circ} \mathrm{C}\right)$, but in the Group 1.1b Thaumarchaeota N. viennensis $\left(37^{\circ} \mathrm{C}\right)$ and $\mathrm{Ca}$. N. gargensis $\left(46^{\circ} \mathrm{C}\right.$; Table S1, Fig. S2). These three species each contain high abundances of specific lipids with unknown properties (e.g., GTGTs, crenarchaeol regioisomer; Fig. 4, Table S1), suggesting that membrane lipid adjustment strategies may vary greatly between thaumarchaeal phylotypes. Analogously to temperature, the high abundance of GDGT-4 in Ca. N. devanaterra has previously been suggested to reflect acclimatization to low $\mathrm{pH}$ by lowering proton permeability (Lehtovirta-Morley et al., 2016). However, GDGT-4 is similarly abundant 
in the neutrophile $N$. viennensis (Fig 4a), indicating that high GDGT-4 abundance may not represent a specific response to low $\mathrm{pH}$. Still, the ring indices of $\mathrm{Ca}$. N. devanaterra are higher than those of the investigated, neutrophilic 1.1a Thaumarchaeota (Table 1), suggesting that like in other archaea, an increase in overall GDGT cycloalkylation in Thaumarchaeota is a common membrane modification mechanism to minimize permeability to protons and other ions in response to a number of physico-chemical and physiological stressors (temperature, $\mathrm{pH}$, energy limitation).

On the phylum level, temperature appears to be a defining parameter of the thaumarchaeal lipidome: Simpson Diversity indices calculated from the relative abundances of all core lipidheadgroup combinations are inversely correlated with (optimal) growth temperatures (Table, Fig. S2 and S4b). Further, controlling for either $\mathrm{pH}$ or temperature during CCA (Table S6, S7) showed that both factors could explain similar amounts of variance, suggesting that both factors may simultaneously influence lipid diversity, although not linearly. This relationship is analogous to inverse correlations between genome size and optimal growth temperature (Sabath et al., 2013). The causality behind genomic size reduction in thermophiles remains unclear, but may be related to reduced cell size and associated reduced maintenance energy expenditure (Sabath et al., 2013; Giovannoni et al., 2014). Similarly, requirements for membrane stability and optimized proton permeability may constrain lipid diversity of Thaumarchaeota (cf. Valentine, 2007) and may represent a phenotypic expression of genomic streamlining. Alternatively, the correlation may represent an artifact resulting either from the phylogenetic dependency of the strains studied here or from the differential genomic capabilities to biosynthesize lipids such as OH-GDGTs, which may have evolved or may have been lost after divergence of the major thaumarchaeal clades. Further research into the relationship between lipid diversity and temperature should include multiple, yet-to-beisolated thermophiles from all major thaumarchaeal clades and could be expanded by including other archaeal phyla, bacteria, or environmental samples.

In contrast to other archaea (e.g., Langworthy, 1977; Shimada et al., 2002; Koga and Morii, 2005; Meador et al., 2014a; Becker et al., 2016), the phylum Thaumarchaeota exhibits very 
low polar headgroup diversity. Further, all studied Thaumarchaeota predominantly synthesize glycosidic rather than glycophosphatidic and phosphatidic headgroups, although all cultures were grown with sufficient phosphate. Collectively, these characteristics indicate that Thaumarchaeota have a reduced biosynthetic demand for phosphorous and are thus well adapted to oligotrophic conditions. This hypothesis is supported by the observation that glycosidic GDGTs predominate over phosphatidic GDGTs in the marine water column (Schouten et al., 2012; Basse et al., 2014; Xie et al., 2014).

Several key differences in membrane lipid composition between the major thaumarchaeal clades may represent additional, ecosystem-specific adaptation mechanisms. For instance, MeO-AR abundance is non-linearly related to temperature across the major phylogenetic clades (Fig. S2). Incorporation of the apolar lipid MeO-AR into the membrane of mesophilic Thaumarchaeota might represent a mechanism for increasing membrane fluidity at low temperatures in a similar way as squalene may increase membrane fluidity in halophilic archaea by spacing polar lipids further apart (Lanyi, 1974; Lanyi et al., 1974). Similarly, based on molecular dynamics modeling (Huguet et al., 2017) and the observation of high relative abundances in high-latitudes (Huguet et al., 2013), OH-GDGTs have been proposed to increase membrane fluidity in marine planktonic Thaumarchaeota. While relative abundances of OH-GDGTs vary between the strains, they are not related to temperature either across the strains studied here (Fig. S4, S9) or in single strains grown at temperatures between $18{ }^{\circ} \mathrm{C}$ and $35^{\circ} \mathrm{C}$ (Elling et al., 2015). Thus, the correlations between temperature and $\mathrm{OH}-\mathrm{GDGT}$ abundance apparent in environmental data could relate to complex effects such as latitudinal shifts in community composition or could be limited to yet uncultured psychrophilic Thaumarchaeota. Thus, membrane regulation mechanisms in Thaumarchaeota appear to be more complex than previously thought and may involve many additional lipids with unresolved properties, such as OH-GDGTs, MeO-AR, GTGTs, GDDs and the crenarchaeol regioisomer.

\section{Experimental procedures}




\section{Cultivation and lipid extraction}

Ca. Nitrosopumilus maritimus strain SCM1 (origin: Seattle Aquarium, USA; Könneke et al., 2005) and Nitrosopumilus spp. strains NAOA2 and NAOA6 pure cultures (Elling et al., 2015) were grown in duplicate at $28{ }^{\circ} \mathrm{C}$ in $8.5 \mathrm{I}$ of $\mathrm{pH} 7.5$ HEPES-buffered SCM medium supplemented with $1.5 \mathrm{mM} \mathrm{NH}_{4} \mathrm{Cl}$ as described previously (Könneke et al., 2005; MartensHabbena et al., 2009). Cultures were harvested in early stationary phase.

Ca. Nitrosopumilus piranensis strain D3C and Ca. Nitrosopumilus adriaticus strain NF5 enrichment cultures (Northern Adriatic Sea surface water; Bayer et al., 2016), were grown at $30^{\circ} \mathrm{C}$ in $2 \mathrm{I}$ of $\mathrm{pH} 7.2 \mathrm{HEPES}$-buffered SCM supplemented with $1 \mathrm{mM} \mathrm{NH}_{4} \mathrm{Cl}$ and harvested in late exponential phase.

A Ca. Nitrososphaera gargensis strain Ga9.2 enrichment culture (Garga hot spring, Russia; Hatzenpichler et al., 2008) was grown at $35^{\circ} \mathrm{C}$ and $46{ }^{\circ} \mathrm{C}$ in $5 \mathrm{I}$ of a pH 7.8 freshwater medium (1 $\mathrm{mM} \mathrm{NH}_{4} \mathrm{Cl}$ ) as described previously (Elling et al., 2016) and harvested in stationary phase.

Nitrososphaera viennensis strains EN76 (DSM 26422) pure and EN123 enrichment cultures (Tourna et al., 2011) were grown at $37^{\circ} \mathrm{C}$ in 15-I batch cultures in pH 7.5 HEPES-buffered freshwater medium modified from Tourna et al. (Tourna et al., 2011) by addition of $1.5 \mathrm{mM}$ pyruvate and $3 \mathrm{mM} \mathrm{NH}_{4} \mathrm{Cl}$ and slight stirring (150 rpm). N. viennensis biomass was harvested in growth phase.

A Ca. Nitrosotalea devanaterra Nd1 pure culture (acidic soil, Aberdeen, UK; LehtovirtaMorley et al., 2014) was grown in batch culture at $25^{\circ} \mathrm{C}$ and $\mathrm{pH} 5.4$ in a synthetic medium $\left(0.5 \mathrm{mM} \mathrm{NH}_{4} \mathrm{Cl}\right)$ modified from Lehtovirta-Morley et al. (2011) by addition of $0.08 \mathrm{~g} \mathrm{I}^{-1}$ of casamino acids and $1 \mu \mathrm{M}$ phthalate buffer solution. Ca. N. devanaterra biomass was harvested in stationary phase.

A Ca. Nitrosocaldus yellowstonii strain HL72 enrichment culture (hot spring, Yellowstone National Park, USA; de la Torre et al., 2008) was grown in batch culture at $72{ }^{\circ} \mathrm{C}$ and $\mathrm{pH} \sim 7$ 
in $3 \mathrm{I}$ of a synthetic freshwater medium ( $1 \mathrm{mM} \mathrm{NH}_{4} \mathrm{Cl}$ ), modified from de la Torre et al. (2008) by addition of $1 \mathrm{mM} \mathrm{pH} 7.5$ MOPS buffer, with a headspace of $80 \% \mathrm{~N}_{2}, 20 \% \mathrm{CO}_{2}$, and $2.5 \mathrm{ml}$ of $100 \% \mathrm{O}_{2}$. Ca. N. yellowstonii biomass was harvested in late growth phase.

Ca. N. maritimus, strains NAOA2 and NAOA6, and Ca. N. gargensis cultures were harvested using a Sartocon Slice cross-flow filtration system (Sartorius, Göttingen, Germany) and subsequent centrifugation (Könneke et al., 2014). Ca. N. devanaterra, N. viennensis, Ca. N. piranensis, Ca. N. adriaticus, and Ca. N. yellowstonii were harvested by centrifugation and subsequently lyophilized.

Cultivation procedures of crenarchaeal and euryarchaeal species are described in Elling et al. (2016).

The cell pellets were stored at $-20{ }^{\circ} \mathrm{C}$ until extraction. Lipids from each batch were extracted following a modified Bligh \& Dyer protocol (Sturt et al., 2004) using a monophasic mixture of methanol, dichloromethane, and aqueous buffer (2:1:0.8, v:v:v) and an ultrasonic probe (15 min sonication; HD2200, Bandelin Electronic, Berlin, Germany). A 50 mM phosphate buffer $(\mathrm{pH}$ 7.4) was used for the first two extractions while a $50 \mathrm{mM}$ trichloroacetic acid buffer $(\mathrm{pH}$ 2) was used for two additional extractions as described by Sturt et al. (2004).

\section{Intact polar and core lipid analysis}

Intact polar and core lipids were quantified by injecting $10-20 \%$ of the total lipid extract (TLE) dissolved in methanol on a Dionex Ultimate 3000 ultra-high performance liquid chromatography (UPLC) system connected to a Bruker maXis Ultra-High Resolution quadrupole time-of-flight tandem mass spectrometer (MS) equipped with an electrospray ionization (ESI) ion source operating in positive mode (Bruker Daltonik, Bremen, Germany). The MS was set to a resolving power of 27,000 at $m / z \quad 1,222$ and each analysis was masscalibrated by loop injections of a calibration standard and correction by lock mass, leading to a mass accuracy of typically less than 1 ppm (Becker et al., 2013; Zhu et al., 2013). Ion source and other MS parameters were optimized by infusion of standards (GDGT-0, 1GGDGT-0, 2G-GDGT-0) into the eluent flow from the UPLC system using a T-piece. 
Analyte separation was achieved using reversed phase UPLC on an ACE3 $\mathrm{C}_{18}$ column $(2.1 \times$ 150 mm, $3 \mu \mathrm{m}$ particle size, Advanced Chromatography Technologies, Aberdeen, Scotland) maintained at $45^{\circ} \mathrm{C}$ as described previously (Zhu et al., 2013).

To determine abundances of core lipid structures relative to total lipids as well as ring index and TEX $\mathrm{T}_{86}$ of total GDGTs, $10 \%$ of the TLE was hydrolyzed with $1 \mathrm{M} \mathrm{HCl}$ in methanol at 70 ${ }^{\circ} \mathrm{C}$ for $3 \mathrm{~h}$ to yield core lipids (Elling et al., 2014). The hydrolyzed TLE was then analyzed on the same UPLC-MS system under different chromatographic conditions using normal phase separation and positive mode atmospheric pressure chemical ionization (APCI) as described previously (Becker et al., 2013).

Lipids were identified by retention time as well as accurate molecular mass and isotope pattern match of proposed sum formulas in full scan mode and $\mathrm{MS}^{2}$ fragment spectra. Integration of peaks was performed on extracted ion chromatograms of $\pm 10 \mathrm{mDa}$ width and included the $[\mathrm{M}+\mathrm{H}]^{+}$ions for normal phase UPLC-MS and additionally $\left[\mathrm{M}+\mathrm{NH}_{4}\right]^{+}$and $[\mathrm{M}+\mathrm{Na}]^{+}$ ions for reversed phase UPLC-MS. Where applicable, double charged ions were included in the integration.

Lipid abundances were corrected for response factors of commercially available as well as purified standards as described previously (Elling et al., 2014). GDGT isomers were summed for calculating relative abundances and ring index, while $\operatorname{TEX}_{86}{ }^{\mathrm{H}}$ was calculated using only the isomer that is dominant in marine sediments, as recommended by (Schouten et al., 2009). The $\operatorname{TEX}_{86}{ }^{\mathrm{H}}$ index was calculated after Schouten et al. (2002) and Kim et al. (2010) using the peak areas of GDGT-1, GDGT-2, GDGT-3 and crenarchaeol regioisomer (Cren'), with the digit indicating the number of cycloalkyl moieties:

$\mathrm{TEX}_{86}^{\mathrm{H}}=\log \frac{[\mathrm{GDGT}-2]+[\mathrm{GDGT}-3]+[\text { Cren'] }}{[\text { GDGT-1]+[GDGT-2]+[GDGT-3]+[Cren'] }}$

TEX ${ }_{86}{ }^{\mathrm{H}}$ reconstructed temperatures were calculated using the core-top calibration of Kim et al. (2010) recommended for temperatures above $15^{\circ} \mathrm{C}\left(\operatorname{TEX}_{86}{ }^{\mathrm{H}}\right)$ :

$\mathrm{SST}=68.4 \times \mathrm{TEX}_{86}^{\mathrm{H}}+38.6$ 
To evaluate GDGT cyclization, we calculated the ring index (RI) according to Pearson et al. (2004):

$\mathrm{RI}=\frac{[\mathrm{GDGT}-1]+2 \times[\text { GDGT-2}]+3 \times[\text { GDGT- }-3]+4 \times[\text { GDGT- }-4]+5 \times\left[\text { Cren }+ \text { Cren }^{\prime}\right]}{[\text { GDGT- }-0]+[\text { GDGT-1 }]+[\text { GDGT-2 }]+[\text { GDGT-3 }]+[\text { GDGT- } 4]+\left[\text { Cren }+ \text { Cren }^{\prime}\right]}$

Phylogenetic analysis

16S rRNA gene sequences were aligned using ClustalW implemented in BioEdit Sequence Alignment Editor (Hall, 1999) before removing regions of ambiguous alignment, leaving 1133 positions. Phylogenetic analyses were performed using General Time Reversible-corrected maximum-likelihood (PhyML, Guindon and Gascuel, 2003), parsimony (MEGA5, Tamura et al., 2011) and Tamura's 3-parameter pairwise distance analysis (MEGA5). Where appropriate, analyses used estimated variable sites only with gamma-distributed site variation and bootstrap support for all methods was calculated 1000 times.

\section{Statistical analyses}

Cluster analyses were performed on the relative abundances of core lipids (after hydrolysis) and intact polar lipids (all core lipid-headgroup combinations including individual cyclized GDGTs) in Matlab R2012b using a Euclidean distance metric and average distance linking. Non-metric multidimensional scaling, constrained correspondence, and redundancy analyses were performed in $R$ (version 3.3.1; R Core Team, 2013) using the vegan package (version 2.4.2; Oksanen et al., 2017). Independent variables (temperature, $\mathrm{pH}$, salinity) were $z$-score standardized for constrained correspondence and redundancy analyses. Lipid relative abundances or lipid indices were used as dependent variables for all statistical analyses. Significance of results from constrained correspondence analyses was tested using the anova function of the vegan package.

Simpson Diversity indices (D) were calculated after Simpson (1949) using relative abundances of core lipid-headgroup combinations for each strain (Meador et al., 2014a):

$D=1-\sum_{n=1}^{118}(\text { relative abundance })^{2}$ 
The value of the Simpson Diversity Index ranges from 0 (no diversity) to 1 (high diversity).

\section{Acknowledgements}

The authors thank the two anonymous reviewers for providing valuable comments that helped improve an earlier version of this manuscript. We thank J.S. Lipp and L.P. Wörmer for assistance with UPLC-MS analysis. R. Bittner (University of Vienna), J. Ross (University of Aberdeen), and V. Russell (San Francisco State University) are thanked for assistance with cultivation and lipid analysis. We thank M.Y. Kellermann for providing extracts of $H$. volcanii and $H$. lacusprofundi. The study was funded by the Deutsche Forschungsgemeinschaft through the Gottfried Wilhelm Leibniz Prize awarded to K.-U. Hinrichs (Hi 616-14-1) and grant Inst 144/300-1 (LC-qToF system), and the Heisenberg fellowship awarded to M.

Könneke (KO 3651/3-1). Procedures for the analysis of lipids were implemented through research funded by the European Research Council under the European Union's Seventh Framework Programme-“Ideas”, ERC grant agreement No. 247153 (Advanced Grant DARCLIFE; PI: K.-U.H.). B. Bayer and G.J. Herndl were supported by the Austrian Science Fund (FWF) project: 1486-B09 and the European Research Council under the European Community's Seventh Framework Program (FP7/2007-2013)/ERC grant agreement No. 268595 (MEDEA project) to GJH. M. Stieglmeier was supported by the Austrian Science Fund (FWF) project P25369-B22 granted to CS.

\section{References}

Auguet, J.-C., Barberan, A., and Casamayor, E.O. (2010) Global ecological patterns in uncultured Archaea. ISME J. 4: 182-190.

Auguet, J.-C. and Casamayor, E.O. (2013) Partitioning of Thaumarchaeota populations along environmental gradients in high mountain lakes. FEMS Microbiol. Ecol. 84: 154164.

Auguet, J.C. and Casamayor, E.O. (2008) A hotspot for cold crenarchaeota in the neuston of high mountain lakes. Environ. Microbiol. 10: 1080-1086.

Baker-Austin, C. and Dopson, M. (2007) Life in acid: pH homeostasis in acidophiles. Trends Microbiol. 15: 165-171. 
Basse, A., Zhu, C., Versteegh, G.J.M., Fischer, G., Hinrichs, K.-U., and Mollenhauer, G. (2014) Distribution of intact and core tetraether lipids in water column profiles of suspended particulate matter off Cape Blanc, NW Africa. Org. Geochem. 72: 1-13.

Bauersachs, T., Weidenbach, K., Schmitz, R. a., and Schwark, L. (2015) Distribution of glycerol ether lipids in halophilic, methanogenic and hyperthermophilic archaea. Org. Geochem. 83-84: 101-108.

Bayer, B., Vojvoda, J., Offre, P., Alves, R.J.E., Elisabeth, N.H., Garcia, J. AL, et al. (2016) Physiological and genomic characterization of two novel marine thaumarchaeal strains indicates niche differentiation. ISME J. 10: 1051-1063.

Becker, K.W., Elling, F.J., Yoshinaga, M.Y., Söllinger, A., Urich, T., and Hinrichs, K.-U. (2016) Unusual Butane- and Pentanetriol-Based Tetraether Lipids in Methanomassiliicoccus luminyensis, a Representative of the Seventh Order of Methanogens. Appl. Environ. Microbiol. 82: 4505-4516.

Becker, K.W., Lipp, J.S., Zhu, C., Liu, X.-L., and Hinrichs, K.-U. (2013) An improved method for the analysis of archaeal and bacterial ether core lipids. Org. Geochem. 61: 34-44.

Biddle, J.F., Lipp, J.S., Lever, M.A., Lloyd, K.G., Sørensen, K.B., Anderson, R., et al. (2006) Heterotrophic Archaea dominate sedimentary subsurface ecosystems off Peru. Proc. Natl. Acad. Sci. U. S. A. 103: 3846-3851.

Bintrim, S.B., Donohue, T.J., Handelsman, J., Roberts, G.P., and Goodman, R.M. (1997) Molecular phylogeny of Archaea from soil. Proc. Natl. Acad. Sci. 94: 277-282.

Boyd, E.S., Pearson, A., Pi, Y., Li, W.-J., Zhang, Y.G., He, L., et al. (2011) Temperature and $\mathrm{pH}$ controls on glycerol dibiphytanyl glycerol tetraether lipid composition in the hyperthermophilic crenarchaeon Acidilobus sulfurireducens. Extremophiles 15: 59-65.

Brochier-Armanet, C., Boussau, B., Gribaldo, S., and Forterre, P. (2008) Mesophilic Crenarchaeota: proposal for a third archaeal phylum, the Thaumarchaeota. Nat. Rev. Microbiol. 6: 245-252.

Chong, P.L.-G. (2010) Archaebacterial bipolar tetraether lipids: Physico-chemical and membrane properties. Chem. Phys. Lipids 163: 253-265.

Coffinet, S., Huguet, A., Williamson, D., Bergonzini, L., Anquetil, C., Majule, A., and Derenne, S. (2015) Occurrence and distribution of glycerol dialkanol diethers and glycerol dialkyl glycerol tetraethers in a peat core from SW Tanzania. Org. Geochem. 83-84: 170-177.

Coolen, M.J.L., Abbas, B., van Bleijswijk, J., Hopmans, E.C., Kuypers, M.M.M., Wakeham, S.G., and Sinninghe Damsté, J.S. (2007) Putative ammonia-oxidizing Crenarchaeota in suboxic waters of the Black Sea: a basin-wide ecological study using $16 \mathrm{~S}$ ribosomal and functional genes and membrane lipids. Environ. Microbiol. 9: 1001-1016.

DeLong, E.F. (1998) Everything in moderation: Archaea as "non-extremophiles." Curr. Opin. Genet. Dev. 8: 649-654.

DeLong, E.F., Wu, K.Y., Prézelin, B.B., and Jovine, R. V (1994) High abundance of Archaea in Antarctic marine picoplankton. Nature 371: 695-697.

Dodsworth, J.A., Hungate, B.A., and Hedlund, B.P. (2011) Ammonia oxidation, denitrification and dissimilatory nitrate reduction to ammonium in two US Great Basin hot springs with abundant ammonia-oxidizing archaea. Environ. Microbiol. 13: 2371-2386.

Elling, F.J., Becker, K.W., Könneke, M., Schröder, J.M., Kellermann, M.Y., Thomm, M., and Hinrichs, K.-U. (2016) Respiratory quinones in Archaea: phylogenetic distribution and 
application as biomarkers in the marine environment. Environ. Microbiol. 18: 692-707.

Elling, F.J., Könneke, M., Lipp, J.S., Becker, K.W., Gagen, E.J., and Hinrichs, K.-U. (2014) Effects of growth phase on the membrane lipid composition of the thaumarchaeon Nitrosopumilus maritimus and their implications for archaeal lipid distributions in the marine environment. Geochim. Cosmochim. Acta 141: 579-597.

Elling, F.J., Könneke, M., Mußmann, M., Greve, A., and Hinrichs, K.-U. (2015) Influence of temperature, $\mathrm{pH}$, and salinity on membrane lipid composition and $\mathrm{TEX}_{86}$ of marine planktonic thaumarchaeal isolates. Geochim. Cosmochim. Acta 171: 238-255.

Feyhl-Buska, J., Chen, Y., Jia, C., Wang, J., Zhang, C.L., and Boyd, E.S. (2016) Influence of Growth Phase, $\mathrm{pH}$, and Temperature on the Abundance and Composition of Tetraether Lipids in the Thermoacidophile Picrophilus torridus. Front. Microbiol. 7: 1323.

Francis, C.A., Roberts, K.J., Beman, J.M., Santoro, A.E., and Oakley, B.B. (2005) Ubiquity and diversity of ammonia-oxidizing archaea in water columns and sediments of the ocean. Proc. Natl. Acad. Sci. U. S. A. 102: 14683-14688.

Giovannoni, S.J., Cameron Thrash, J., and Temperton, B. (2014) Implications of streamlining theory for microbial ecology. ISME J. 8: 1-13.

Gubry-Rangin, C., Hai, B., Quince, C., Engel, M., Thomson, B.C., James, P., et al. (2011) Niche specialization of terrestrial archaeal ammonia oxidizers. Proc. Natl. Acad. Sci. U. S. A. 108: 21206-21211.

Guindon, S. and Gascuel, O. (2003) A Simple, Fast, and Accurate Algorithm to Estimate Large Phylogenies by Maximum Likelihood. Syst. Biol. 52: 696-704.

Hall, T.A. (1999) BioEdit: a user-friendly biological sequence alignment editor and analysis program for Windows 95/98/NT. Nucleic Acids Symp. Ser. 41: 95-98.

Hatzenpichler, R., Lebedeva, E. V, Spieck, E., Stoecker, K., Richter, A., Daims, H., and Wagner, M. (2008) A moderately thermophilic ammonia-oxidizing crenarchaeote from a hot spring. Proc. Natl. Acad. Sci. U. S. A. 105: 2134-2139.

Hinrichs, K.-U., Hayes, J.M., Sylva, S.P., Brewer, P.G., and DeLong, E.F. (1999) Methaneconsuming archaebacteria in marine sediments. Nature 398: 802-805.

Hopmans, E.C., Schouten, S., and Sinninghe Damsté, J.S. (2016) The effect of improved chromatography on GDGT-based palaeoproxies. Org. Geochem. 93: 1-6.

Huguet, C., Fietz, S., and Rosell-Melé, A. (2013) Global distribution patterns of hydroxy glycerol dialkyl glycerol tetraethers. Org. Geochem. 57: 107-118.

Huguet, C., Fietz, S., Rosell-Melé, A., Daura, X., Costenaro, L.-O., and Costenaro, L. (2017) Molecular dynamics simulation study of the effect of glycerol dialkyl glycerol tetraether hydroxylation on membrane thermostability. BBA - Biomembr.

Hurley, S.J., Elling, F.J., Könneke, M., Buchwald, C., Wankel, S.D., Santoro, A.E., et al. (2016) Influence of ammonia oxidation rate on thaumarchaeal lipid composition and the TEX $_{86}$ temperature proxy. Proc. Natl. Acad. Sci. 113: 7762-7767.

Jung, M.-Y., Park, S.-J., Min, D., Kim, J.-S., Rijpstra, W.I.C., Sinninghe Damsté, J.S., et al. (2011) Enrichment and characterization of an autotrophic ammonia-oxidizing archaeon of mesophilic crenarchaeal group I.1a from an agricultural soil. Appl. Environ. Microbiol. 77: 8635-8647.

Karner, M.B., DeLong, E.F., and Karl, D.M. (2001) Archaeal dominance in the mesopelagic 
zone of the Pacific Ocean. Nature 409: 507-510.

Kim, J.-H., van der Meer, J., Schouten, S., Helmke, P., Willmott, V., Sangiorgi, F., et al. (2010) New indices and calibrations derived from the distribution of crenarchaeal isoprenoid tetraether lipids: Implications for past sea surface temperature reconstructions. Geochim. Cosmochim. Acta 74: 4639-4654.

Knappy, C., Barillà, D., Chong, J., Hodgson, D., Morgan, H., Suleman, M., et al. (2015) Mono- , di- and trimethylated homologues of isoprenoid tetraether lipid cores in archaea and environmental samples : mass spectrometric identification and significance. 14201432.

Knappy, C.S. and Keely, B.J. (2012) Novel glycerol dialkanol triols in sediments: transformation products of glycerol dibiphytanyl glycerol tetraether lipids or biosynthetic intermediates? Chem. Commun. 48: 841-843.

Knappy, C.S., Nunn, C.E.M., Morgan, H.W., and Keely, B.J. (2011) The major lipid cores of the archaeon Ignisphaera aggregans: implications for the phylogeny and biosynthesis of glycerol monoalkyl glycerol tetraether isoprenoid lipids. Extremophiles 15: 517-528.

Koga, Y. and Morii, H. (2005) Recent advances in structural research on ether lipids from Archaea including comparative and physiological aspects. Biosci. Biotechnol. Biochem. 69: 2019-2034.

Konings, W.N., Albers, S.-V., Koning, S., and Driessen, A.J.M. (2002) The cell membrane plays a crucial role in survival of bacteria and archaea in extreme environments. Antonie Van Leeuwenhoek 81: 61-72.

Könneke, M., Bernhard, A.E., de la Torre, J.R., Walker, C.B., Waterbury, J.B., and Stahl, D.A. (2005) Isolation of an autotrophic ammonia-oxidizing marine archaeon. Nature 437: 543-546.

Könneke, M., Schubert, D.M., Brown, P.C., Hügler, M., Standfest, S., Schwander, T., et al. (2014) Ammonia-oxidizing archaea use the most energy-efficient aerobic pathway for $\mathrm{CO}_{2}$ fixation. Proc. Natl. Acad. Sci. U. S. A. 111: 8239-8244.

de la Torre, J.R., Walker, C.B., Ingalls, A.E., Könneke, M., and Stahl, D.A. (2008) Cultivation of a thermophilic ammonia oxidizing archaeon synthesizing crenarchaeol. Environ. Microbiol. 10: 810-818.

Langworthy, T.A. (1977) Comparative lipid composition of heterotrophically and autotrophically grown Sulfolobus acidocaldarius. J. Bacteriol. 130: 1326-1332.

Lanyi, J.K. (1974) Irregular bilayer structure in vesicles prepared from Halobacterium cutirubrum lipids. Biochim. Biophys. Acta 356: 245-256.

Lanyi, J.K., Plachy, W.Z., and Kates, M. (1974) Lipid interactions in membranes of extremely halophilic bacteria. II. Modification of the bilayer structure by squalene. Biochemistry 13: 4914-4920.

Lebedeva, E. V, Hatzenpichler, R., Pelletier, E., Schuster, N., Hauzmayer, S., Bulaev, A., et al. (2013) Enrichment and genome sequence of the group I.1a ammonia-oxidizing archaeon " $\mathrm{Ca}$. Nitrosotenuis uzonensis" representing a clade globally distributed in thermal habitats. PLoS One 8: e80835.

Lehtovirta-Morley, L.E., Ge, C., Ross, J., Yao, H., Nicol, G.W., and Prosser, J.I. (2014) Characterisation of terrestrial acidophilic archaeal ammonia oxidisers and their inhibition and stimulation by organic compounds. FEMS Microbiol. Ecol. 89: 542-552. 
Lehtovirta-Morley, L.E., Sayavedra-Soto, L.A., Gallois, N., Schouten, S., Stein, L.Y., Prosser, J.I., and Nicol, G.W. (2016) Identifying potential mechanisms enabling acidophily in the ammonia-oxidising archaeon "Candidatus Nitrosotalea devanaterra." Appl. Environ. Microbiol. 44: AEM.04031-15.

Lehtovirta-Morley, L.E., Stoecker, K., Vilcinskas, A., Prosser, J.I., and Nicol, G.W. (2011) Cultivation of an obligate acidophilic ammonia oxidizer from a nitrifying acid soil. Proc. Natl. Acad. Sci. U. S. A. 108: 15892-15897.

Lehtovirta, L.E., Prosser, J.I., and Nicol, G.W. (2009) Soil pH regulates the abundance and diversity of Group 1.1c Crenarchaeota. FEMS Microbiol. Ecol. 70: 367-376.

Leininger, S., Urich, T., Schloter, M., Schwark, L., Qi, J., Nicol, G.W., et al. (2006) Archaea predominate among ammonia-oxidizing prokaryotes in soils. Nature 442: 806-809.

Lincoln, S.A., Wai, B., Eppley, J.M., Church, M.J., Summons, R.E., and DeLong, E.F. (2014a) Planktonic Euryarchaeota are a significant source of archaeal tetraether lipids in the ocean. Proc. Natl. Acad. Sci. U. S. A. 111: 9858-9863.

Lincoln, S.A., Wai, B., Eppley, J.M., Church, M.J., Summons, R.E., and DeLong, E.F. (2014b) Reply to Schouten et al.: Marine Group II planktonic Euryarchaeota are significant contributors to tetraether lipids in the ocean. Proc. Natl. Acad. Sci. U. S. A. 111: 4286.

Liu, X.-L., Birgel, D., Elling, F.J., Sutton, P.A., Lipp, J.S., Zhu, R., et al. (2016) From ether to acid: A plausible degradation pathway of glycerol dialkyl glycerol tetraethers. Geochim. Cosmochim. Acta 183: 138-152.

Liu, X.-L., Lipp, J.S., Schröder, J.M., Summons, R.E., and Hinrichs, K.-U. (2012a) Isoprenoid glycerol dialkanol diethers: A series of novel archaeal lipids in marine sediments. Org. Geochem. 43: 50-55.

Liu, X.-L., Lipp, J.S., Simpson, J.H., Lin, Y.-S., Summons, R.E., and Hinrichs, K.-U. (2012b) Mono- and dihydroxyl glycerol dibiphytanyl glycerol tetraethers in marine sediments: Identification of both core and intact polar lipid forms. Geochim. Cosmochim. Acta 89: 102-115.

Liu, X.-L., Summons, R.E., and Hinrichs, K.-U. (2012c) Extending the known range of glycerol ether lipids in the environment: structural assignments based on tandem mass spectral fragmentation patterns. Rapid Commun. Mass Spectrom. 26: 2295-2302.

Logemann, J., Graue, J., Köster, J., Engelen, B., Rullkötter, J., and Cypionka, H. (2011) A laboratory experiment of intact polar lipid degradation in sandy sediments. Biogeosciences 8: 2547-2560.

Martens-Habbena, W., Berube, P.M., Urakawa, H., de la Torre, J.R., and Stahl, D.A. (2009) Ammonia oxidation kinetics determine niche separation of nitrifying Archaea and Bacteria. Nature 461: 976-979.

Mathai, J.C., Sprott, G.D., and Zeidel, M.L. (2001) Molecular mechanisms of water and solute transport across archaebacterial lipid membranes. J. Biol. Chem. 276: 2726627271.

Meador, T.B., Gagen, E.J., Loscar, M.E., Goldhammer, T., Yoshinaga, M.Y., Wendt, J., et al. (2014a) Thermococcus kodakarensis modulates its polar membrane lipids and elemental composition according to growth stage and phosphate availability. Front. Microbiol. 5: 10. 
Meador, T.B., Zhu, C., Elling, F.J., Könneke, M., and Hinrichs, K.-U. (2014b) Identification of isoprenoid glycosidic glycerol dibiphytanol diethers and indications for their biosynthetic origin. Org. Geochem. 69: 70-75.

Murray, A.E., Preston, C.M., Massana, R., Taylor, L.T., Blakis, A., Wu, K., and DeLong, E.F. (1998) Seasonal and spatial variability of bacterial and archaeal assemblages in the coastal waters near Anvers Island, Antarctica. Appl. Environ. Microbiol. 64: 2585-2595.

Ngugi, D.K., Blom, J., Alam, I., Rashid, M., Ba-Alawi, W., Zhang, G., et al. (2015) Comparative genomics reveals adaptations of a halotolerant thaumarchaeon in the interfaces of brine pools in the Red Sea. ISME J. 9: 396-411.

Nicol, G.W., Leininger, S., Schleper, C., and Prosser, J.I. (2008) The influence of soil pH on the diversity, abundance and transcriptional activity of ammonia oxidizing archaea and bacteria. Environ. Microbiol. 10: 2966-2978.

Nicol, G.W. and Schleper, C. (2006) Ammonia-oxidising Crenarchaeota: important players in the nitrogen cycle? Trends Microbiol. 14: 207-212.

Ochsenreiter, T., Selezi, D., Quaiser, A., Bonch-Osmolovskaya, L., and Schleper, C. (2003) Diversity and abundance of Crenarchaeota in terrestrial habitats studied by 16S RNA surveys and real time PCR. Environ. Microbiol. 5: 787-797.

Oger, P.M. and Cario, A. (2013) Adaptation of the membrane in Archaea. Biophys. Chem. 183: 42-56.

Oksanen, J., Blanchet, F.G., Friendly, M., Kindt, R., Legendre, P., McGlinn, D., et al. (2017) vegan: Community Ecology Package. https://cran.r-project.org/package=vegan.

Pearson, A. (2014) Lipidomics for Geochemistry 2nd ed. Falkowski,P.G. and Freeman,K.H. (eds) Elsevier Ltd., London, UK.

Pearson, A., Huang, Z., Ingalls, A.E., Romanek, C.S., Wiegel, J., Freeman, K.H., et al. (2004) Nonmarine crenarchaeol in Nevada hot springs. Appl. Environ. Microbiol. 70: 5229-5237.

Pearson, A., McNichol, A.P., Benitez-Nelson, B.C., Hayes, J.M., and Eglinton, T.I. (2001) Origins of lipid biomarkers in Santa Monica Basin surface sediment: a case study using compound-specific $\Delta^{14} \mathrm{C}$ analysis. Geochim. Cosmochim. Acta 65: 3123-3137.

Pester, M., Rattei, T., Flechl, S., Gröngröft, A., Richter, A., Overmann, J., et al. (2012) amoAbased consensus phylogeny of ammonia-oxidizing archaea and deep sequencing of amoA genes from soils of four different geographic regions. Environ. Microbiol. 14: 525539.

Pester, M., Schleper, C., and Wagner, M. (2011) The Thaumarchaeota: an emerging view of their phylogeny and ecophysiology. Curr. Opin. Microbiol. 14: 300-306.

Pitcher, A., Hopmans, E.C., Mosier, A.C., Park, S.-J., Rhee, S.-K., Francis, C.A., et al. (2011) Core and intact polar glycerol dibiphytanyl glycerol tetraether lipids of ammoniaoxidizing archaea enriched from marine and estuarine sediments. Appl. Environ. Microbiol. 77: 3468-3477.

Pitcher, A., Rychlik, N., Hopmans, E.C., Spieck, E., Rijpstra, W.I.C., Ossebaar, J., et al. (2010) Crenarchaeol dominates the membrane lipids of Candidatus Nitrososphaera gargensis, a thermophilic group I.1b Archaeon. ISME J. 4: 542-552.

Prosser, J.I. and Nicol, G.W. (2008) Relative contributions of archaea and bacteria to aerobic ammonia oxidation in the environment. Environ. Microbiol. 10: 2931-2941. 
Qin, W., Carlson, L.T., Armbrust, E.V., Devol, A.H., Moffett, J.W., Stahl, D.A., and Ingalls, A.E. (2015) Confounding effects of oxygen and temperature on the $\mathrm{TEX}_{86}$ signature of marine Thaumarchaeota. Proc. Natl. Acad. Sci. 112: 10979-10984.

R Core Team (2013) R: A Language and Environment for Statistical Computing. http://www.r-project.org/.

Ramette, A. (2007) Multivariate analyses in microbial ecology. FEMS Microbiol. Ecol. 62: $142-160$.

Reigstad, L.J., Richter, A., Daims, H., Urich, T., Schwark, L., and Schleper, C. (2008) Nitrification in terrestrial hot springs of Iceland and Kamchatka. FEMS Microbiol. Ecol. 64: 167-174.

De Rosa, M., Esposito, E., Gambacorta, A., Nicolaus, B., and Bu'Lock, J.D. (1980) Effects of temperature on ether lipid composition of Caldariella acidophila. Phytochemistry 19: 827-831.

De Rosa, M. and Gambacorta, A. (1988) The lipids of archaebacteria. Prog. Lipid Res. 27: $153-175$.

De Rosa, M., Gambacorta, A., Nicolaus, B., Chappe, B., and Albrecht, P. (1983) Isoprenoid ethers; backbone of complex lipids of the archaebacterium Sulfolobus solfataricus. Biochim. Biophys. Acta 753: 249-256.

Sabath, N., Ferrada, E., Barve, A., and Wagner, A. (2013) Growth temperature and genome size in bacteria are negatively correlated, suggesting genomic streamlining during thermal adaptation. Genome Biol. Evol. 5: 966-977.

Schattenhofer, M., Fuchs, B.M., Amann, R., Zubkov, M. V, Tarran, G.A., and Pernthaler, J. (2009) Latitudinal distribution of prokaryotic picoplankton populations in the Atlantic Ocean. Environ. Microbiol. 11: 2078-2093.

Schleper, C., Jurgens, G., and Jonuscheit, M. (2005) Genomic studies of uncultivated archaea. Nat. Rev. Microbiol. 3: 479-488.

Schouten, S., Hopmans, E.C., Baas, M., Boumann, H., Standfest, S., Könneke, M., et al. (2008) Intact Membrane Lipids of "Candidatus Nitrosopumilus maritimus," a Cultivated Representative of the Cosmopolitan Mesophilic Group I Crenarchaeota. Appl. Environ. Microbiol. 74: 2433-2440.

Schouten, S., Hopmans, E.C., van der Meer, J., Mets, A., Bard, E., Bianchi, T.S., et al. (2009) An interlaboratory study of $\mathrm{TEX}_{86}$ and BIT analysis using high-performance liquid chromatography-mass spectrometry. Geochemistry, Geophys. Geosystems 10: Q03012.

Schouten, S., Hopmans, E.C., Schefuß, E., and Sinninghe Damsté, J.S. (2002) Distributional variations in marine crenarchaeotal membrane lipids: a new tool for reconstructing ancient sea water temperatures? Earth Planet. Sci. Lett. 204: 265-274.

Schouten, S., Pitcher, A., Hopmans, E.C., Villanueva, L., van Bleijswijk, J., and Sinninghe Damsté, J.S. (2012) Intact polar and core glycerol dibiphytanyl glycerol tetraether lipids in the Arabian Sea oxygen minimum zone: I. Selective preservation and degradation in the water column and consequences for the $\mathrm{TEX}_{86}$. Geochim. Cosmochim. Acta 98: 228-243.

Schouten, S., Villanueva, L., Hopmans, E.C., van der Meer, M.T.J., and Sinninghe Damsté, J.S. (2014) Are Marine Group II Euryarchaeota significant contributors to tetraether 
lipids in the ocean? Proc. Natl. Acad. Sci. U. S. A. 111: 4285.

Schubotz, F., Lipp, J.S., Elvert, M., and Hinrichs, K.-U. (2011) Stable carbon isotopic compositions of intact polar lipids reveal complex carbon flow patterns among hydrocarbon degrading microbial communities at the Chapopote asphalt volcano. Geochim. Cosmochim. Acta 75: 4399-4415.

Schubotz, F., Wakeham, S.G., Lipp, J.S., Fredricks, H.F., and Hinrichs, K.-U. (2009) Detection of microbial biomass by intact polar membrane lipid analysis in the water column and surface sediments of the Black Sea. Environ. Microbiol. 11: 2720-2734.

Shimada, H., Nemoto, N., Shida, Y., Oshima, T., and Yamagishi, A. (2002) Complete Polar Lipid Composition of Thermoplasma acidophilum HO-62 Determined by HighPerformance Liquid Chromatography with Evaporative Light-Scattering Detection. J. Bacteriol. 184: 556-563.

Simpson, E.H. (1949) Measurement of Diversity. Nature 163: 688-688.

Sinninghe Damsté, J.S., Rijpstra, W.I.C., Hopmans, E.C., Jung, M.-Y., Kim, J.-G., Rhee, S.K., et al. (2012) Intact polar and core glycerol dibiphytanyl glycerol tetraether lipids of group I.1a and I.1b Thaumarchaeota in soil. Appl. Environ. Microbiol. 78: 6866-6874.

Sinninghe Damsté, J.S., Schouten, S., Hopmans, E.C., van Duin, A.C.T., and Geenevasen, J.A.J. (2002) Crenarchaeol: the characteristic core glycerol dibiphytanyl glycerol tetraether membrane lipid of cosmopolitan pelagic crenarchaeota. J. Lipid Res. 43: $1641-1651$.

Spang, A., Hatzenpichler, R., Brochier-Armanet, C., Rattei, T., Tischler, P., Spieck, E., et al. (2010) Distinct gene set in two different lineages of ammonia-oxidizing archaea supports the phylum Thaumarchaeota. Trends Microbiol. 18: 331-340.

Sprott, G.D., Agnew, B.J., and Patel, G.B. (1997) Structural features of ether lipids in the archaeobacterial thermophiles Pyrococcus furiosus, Methanopyrus kandleri, Methanothermus fervidus, and Sulfolobus acidocaldarius. Can. J. Microbiol. 43: 467476.

Stahl, D.A. and de la Torre, J.R. (2012) Physiology and Diversity of Ammonia-Oxidizing Archaea. Annu. Rev. Microbiol. 66: 83-101.

Stieglmeier, M., Klingl, A., Alves, R.J.E., Rittmann, S.K.-M.R., Melcher, M., Leisch, N., and Schleper, C. (2014) Nitrososphaera viennensis gen. nov., sp. nov., an aerobic and mesophilic, ammonia-oxidizing archaeon from soil and a member of the archaeal phylum Thaumarchaeota. Int. J. Syst. Evol. Microbiol. 64: 2738-2752.

Sturt, H.F., Summons, R.E., Smith, K., Elvert, M., and Hinrichs, K.-U. (2004) Intact polar membrane lipids in prokaryotes and sediments deciphered by high-performance liquid chromatography/electrospray ionization multistage mass spectrometry - new biomarkers for biogeochemistry and microbial ecology. Rapid Commun. Mass Spectrom. 18: 617628.

Tamura, K., Peterson, D., Peterson, N., Stecher, G., Nei, M., and Kumar, S. (2011) MEGA5: Molecular Evolutionary Genetics Analysis Using Maximum Likelihood, Evolutionary Distance, and Maximum Parsimony Methods. Mol. Biol. Evol. 28: 2731-2739.

Tarui, M., Tanaka, N., Tomura, K., Ohga, M., Morii, H., and Koga, Y. (2007) Lipid component parts analysis of the hyperthermophilic sulfate-reducing archaeon Archaeoglobus fulgidus. J. UOEH 29: 131-139. 
Tourna, M., Stieglmeier, M., Spang, A., Könneke, M., Schintlmeister, A., Urich, T., et al. (2011) Nitrososphaera viennensis, an ammonia oxidizing archaeon from soil. Proc. Natl. Acad. Sci. U. S. A. 108: 8420-8425.

Valentine, D.L. (2007) Adaptations to energy stress dictate the ecology and evolution of the Archaea. Nat. Rev. Microbiol. 5: 316-323.

Villanueva, L., Sinninghe Damsté, J.S., and Schouten, S. (2014) A re-evaluation of the archaeal membrane lipid biosynthetic pathway. Nat. Rev. Microbiol. 12: 438-448.

van de Vossenberg, J.L.C.M., Driessen, A.J.M., and Konings, W.N. (1998) The essence of being extremophilic: the role of the unique archaeal membrane lipids. Extremophiles 2: 163-170.

Van de Vossenberg, J.L.C.M., Driessen, A.J.M., Zillig, W., and Konings, W.N. (1998) Bioenergetics and cytoplasmic membrane stability of the extremely acidophilic, thermophilic archaeon Picrophilus oshimae. Extremophiles 2: 67-74.

Wakeham, S.G., Amann, R., Freeman, K.H., Hopmans, E.C., Jørgensen, B.B., Putnam, I.F., et al. (2007) Microbial ecology of the stratified water column of the Black Sea as revealed by a comprehensive biomarker study. Org. Geochem. 38: 2070-2097.

Wakeham, S.G., Turich, C., Schubotz, F., Podlaska, A., Li, X.N., Varela, R., et al. (2012) Biomarkers, chemistry and microbiology show chemoautotrophy in a multilayer chemocline in the Cariaco Basin. Deep Sea Res. Part I Oceanogr. Res. Pap. 63: 133156.

Walker, C.B., de la Torre, J.R., Klotz, M.G., Urakawa, H., Pinel, N., Arp, D.J., et al. (2010) Nitrosopumilus maritimus genome reveals unique mechanisms for nitrification and autotrophy in globally distributed marine crenarchaea. Proc. Natl. Acad. Sci. U. S. A. 107: 8818-8823.

Wu, W., Ruan, J., Ding, S., Zhao, L., Xu, Y., Yang, H., et al. (2014) Source and distribution of glycerol dialkyl glycerol tetraethers along lower Yellow River-estuary--coast transect. Mar. Chem. 158: 17-26.

Xie, S., Lipp, J.S., Wegener, G., Ferdelman, T.G., and Hinrichs, K.-U. (2013) Turnover of microbial lipids in the deep biosphere and growth of benthic archaeal populations. Proc. Natl. Acad. Sci. U. S. A. 110: 6010-6014.

Xie, S., Liu, X.-L., Schubotz, F., Wakeham, S.G., and Hinrichs, K.-U. (2014) Distribution of glycerol ether lipids in the oxygen minimum zone of the Eastern Tropical North Pacific Ocean. Org. Geochem. 71: 60-71.

Yamauchi, K., Doi, K., Yoshida, Y., and Kinoshita, M. (1993) Archaebacterial lipids: highly proton-impermeable membranes from 1,2-diphytanyl-sn-glycero-3-phosphocoline. Biochim. Biophys. Acta 1146: 178-182.

Yang, H., Pancost, R.D., Tang, C., Ding, W., Dang, X., and Xie, S. (2014) Distributions of isoprenoid and branched glycerol dialkanol diethers in Chinese surface soils and a loess--paleosol sequence: Implications for the degradation of tetraether lipids. Org. Geochem. 66: 70-79.

Zhu, C., Lipp, J.S., Wörmer, L., Becker, K.W., Schröder, J., and Hinrichs, K.-U. (2013) Comprehensive glycerol ether lipid fingerprints through a novel reversed phase liquid chromatography-mass spectrometry protocol. Org. Geochem. 65: 53-62.

Zhu, C., Wakeham, S.G., Elling, F.J., Basse, A., Mollenhauer, G., Versteegh, G.J.M., et al. 
(2016) Stratification of archaeal membrane lipids in the ocean and implications for adaptation and chemotaxonomy of planktonic archaea. Environ. Microbiol.

\section{Figure captions}

Fig. 1. Maximum-likelihood phylogenetic analysis of 16S rRNA genes of organisms analyzed in this study (in bold) combined with other cultivated Thaumarchaeota with sequenced genomes placed with four major AOA lineages. Analyses were performed on 1133 unambiguously aligned positions and values at major nodes represent the most conservative bootstrap support from three methods of analysis (ML, parsimony and distance). The scale bar represents 0.05 changes per nucleotide position.

Fig. 2. Structures of thaumarchaeal glycerol dibiphytanyl glycerol tetraether (GDGT) and glycerol diphytanyl diether (archaeol) core lipids (adapted from Elling et al., 2015). GDGTs may contain up to four cyclopentane rings or one cyclohexane and four cyclopentane rings (crenarchaeol). Derivatives comprise GDGTs containing one (OH-GDGT) or two (2OHGDGT) additional hydroxyl groups and zero to four cyclopentane rings in the biphytanyl side chain, acyclic or monocyclic glycerol trialkyl glycerol tetraether (GTGT), zero to five ringbearing glycerol dialkanol diethers (GDDs) as well as GDGT and archaeol containing a methoxy group at the sn-1 position of the glycerol moiety (MeO-GDGT and MeO-AR). Monounsaturated $\left(\mathrm{MK}_{6: 1}\right)$ and saturated menaquinone-6 $\left(\mathrm{MK}_{6: 0}\right)$ are isoprenoidal membranesoluble electron carriers. Thaumarchaeal intact polar lipids consist of one or two glycosidic or glycophosphatidic headgroups attached to the glycerol sn-1 hydroxyl position of a diether or tetraether core lipid.

Fig. 3. Distribution of 118 lipids among the lipidomes of the four major phylogenetic subgroups of the phylum Thaumarchaeota with cultivated representatives (based on analyses of ten thaumarchaeal cultures).

Fig. 4. Cluster analyses of the relative abundances of $(A)$ major core lipids (including isomers) and (B) intact polar lipid types in ten thaumarchaeal strains ( $N$. gargensis grown at 
$46{ }^{\circ} \mathrm{C}$ ). Phylogenetically closely related strains share high similarity in their core lipid composition, while cultures from similar habitats show close relatedness in their intact polar lipid compositions.

Fig. 5. Distribution of GDGT structural types in the major thaumarchaeal intact polar lipid classes 1G-GDGT, 2G-GDGT, 2G-OH-GDGT, and HPH-GDGT as well as in total GDGTs derived from hydrolysis in ten cultivated thaumarchaeal strains $\left(N\right.$. gargensis grown at $\left.46{ }^{\circ} \mathrm{C}\right)$ as well as average composition for Group 1.1a and 1.1b.

Fig. 6. (A) Extracted ion chromatograms showing elution of GDGT-1, -2, -3, -4, crenarchaeol and their isomers (a, b, c, cren') in a UPLC-APCI-MS analysis of a Nitrosopumilus maritimus total lipid extract harvested in early growth phase (not used for panels B-F, intensity not to scale). Uncolored peaks in each chromatogram represent $+2 \mathrm{Da}$ isotope peaks of the respective lighter GDGT. (B to F) Relative abundances of GDGT-1, -2, -3, -4, and crenarchaeol and their isomers in thaumarchaeal hydrolyzed total lipid extracts as determined using UPLC-APCI-MS (means of duplicate cultures).

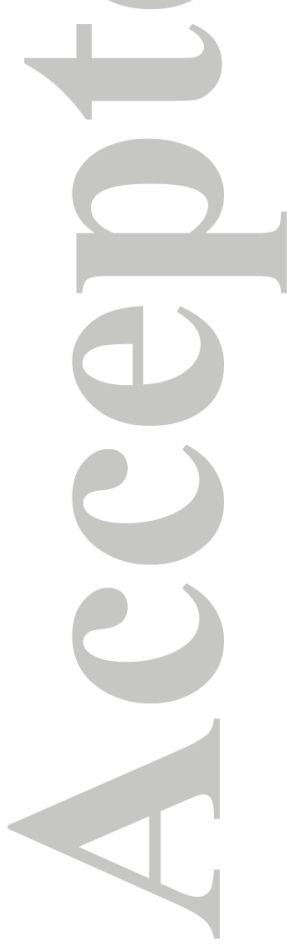


Table 1. Abundances of archaeol (AR), methoxy archaeol (MeO-AR), summed crenarchaeol and regioisomer (cren + cren') in thaumarchaeal cultures relative to total lipids derived by acid hydrolysis as well as GDGT cyclization degree (ring index), $\operatorname{TEX}_{86}{ }^{\mathrm{H}}$ and calculated TEX ${ }_{86}{ }^{\mathrm{H}}$-temperature in total hydrolysis-derived GDGTs (measured using normal phase UPLC-APCI-MS), and growth medium parameters (salinity estimated from total weight of salts added to the medium). Simpson diversity was calculated based on full lipid diversity including individual cyclized core and intact polar GDGTs, respectively, as determined by UPLC-ESI-MS. N/A: not available.

\begin{tabular}{|c|c|c|c|c|c|c|c|c|c|c|c|c|}
\hline 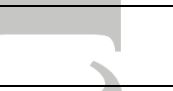 & $\begin{array}{c}\text { Growth } \\
\text { temperature } \\
\left({ }^{\circ} \mathrm{C}\right)\end{array}$ & $\begin{array}{c}\text { Growth } \\
\mathrm{pH}\end{array}$ & $\begin{array}{l}\mathrm{NH}_{4}^{+} \\
(\mathrm{mM})\end{array}$ & $\begin{array}{l}\mathrm{HCO}_{3}^{-} \\
(\mathrm{mM})\end{array}$ & $\begin{array}{c}\text { Salinity } \\
\left(\mathrm{g} \mathrm{l}^{-1}\right)\end{array}$ & $\begin{array}{l}\text { AR } \\
(\%)\end{array}$ & $\begin{array}{c}\mathrm{MeO}- \\
\mathrm{AR} \\
(\%) \\
\end{array}$ & $\begin{array}{c}\text { Cren+ } \\
\text { Cren' } \\
(\%) \\
\end{array}$ & $\begin{array}{l}\text { Simpson } \\
\text { Diversity }\end{array}$ & $\begin{array}{l}\text { Ring } \\
\text { index }\end{array}$ & $\mathrm{TEX}_{86}{ }^{\mathrm{H}}$ & $\begin{array}{c}\text { TEX }_{86}{ }^{\mathrm{H}}{ }^{-} \\
\text {Temp. } \\
\left({ }^{\circ} \mathrm{C}\right)\end{array}$ \\
\hline $\begin{array}{l}\text { Nitrosotalea } \\
\text { devanaterra }\end{array}$ & 25 & 5.4 & 0.5 & 2 & 2.2 & 0.3 & 20.9 & 7.6 & 0.89 & 3.7 & $\begin{array}{c}- \\
0.0458\end{array}$ & 35.5 \\
\hline $\begin{array}{l}\text { Nitrosopumilus } \\
\text { maritimus }\end{array}$ & 28 & 7.5 & 1.5 & 2 & 37 & 0.2 & 2.1 & 11.0 & 0.93 & 3.0 & 0.0969 & 32.0 \\
\hline Strain NAOA2 & 28 & 7.5 & 1.5 & 2 & 37 & 0.6 & 2.3 & 4.7 & 0.91 & 2.9 & $\begin{array}{c}- \\
0.0605\end{array}$ & 34.5 \\
\hline Strain NAOA6 & 28 & 7.5 & 1.5 & 2 & 37 & 0.4 & 4.5 & 5.0 & 0.93 & 2.7 & $\begin{array}{c}- \\
0.0605\end{array}$ & 34.5 \\
\hline $\begin{array}{l}\text { Nitrosopumilus } \\
\text { piranensis }\end{array}$ & 30 & 7.2 & 1 & 2 & 37 & 2.4 & 5.3 & 4.6 & 0.90 & 3.0 & $\begin{array}{c}- \\
0.0862\end{array}$ & 32.5 \\
\hline $\begin{array}{l}\text { Nitrosopumilus } \\
\text { adriaticus }\end{array}$ & 30 & 7.2 & 1 & 2 & 37 & 4.2 & 11.5 & 6.7 & 0.90 & 3.0 & $\begin{array}{c}- \\
0.1079\end{array}$ & 31.2 \\
\hline $\begin{array}{l}\text { Nitrososphaera } \\
\text { viennensis } \\
\text { EN76 }\end{array}$ & 37 & 7.5 & 3 & 2 & 2.2 & 1.5 & 1.9 & 7.6 & 0.85 & 4.3 & $\begin{array}{c}- \\
0.0044\end{array}$ & 38.2 \\
\hline $\begin{array}{l}\text { Nitrososphaera } \\
\text { viennensis } \\
\text { EN123 }\end{array}$ & 37 & 7.5 & 3 & 2 & 2.2 & 0.3 & 1.6 & 8.5 & 0.84 & 4.3 & $\overline{-} . \overline{0}$ & 38.4 \\
\hline $\begin{array}{l}\text { Nitrososphaera } \\
\text { gargensis }\end{array}$ & 35 & 7.8 & 1 & 2 & 10.5 & 2.1 & $<0.1$ & 41.4 & $\mathrm{~N} / \mathrm{A}$ & 4.4 & $\begin{array}{c}- \\
0.0362\end{array}$ & 36.1 \\
\hline $\begin{array}{l}\text { Nitrososphaera } \\
\text { gargensis }\end{array}$ & 46 & 7.8 & 1 & 2 & 10.5 & 1.1 & 0.2 & 60.3 & 0.80 & 4.8 & $\begin{array}{c}- \\
0.0132\end{array}$ & 37.6 \\
\hline $\begin{array}{l}\text { Nitrosocaldus } \\
\text { yellowstonii }\end{array}$ & 72 & 7 & 1 & 1 & 2 & 6.2 & 0.1 & 9.1 & 0.51 & 3.8 & $\begin{array}{c}- \\
0.2147\end{array}$ & 24.1 \\
\hline
\end{tabular}

Wiley-Blackwell and Society for Applied Microbiology

This article is protected by copyright. All rights reserved. 
Table 2. Phylogenetic occurrence (+ presence; - absence) of archaeol (AR), hydroxyarchaeol (OH-AR), methoxy archaeol (MeO-AR), GDGTs (numbers indicate presence of ring-containing GDGTs), GTGTs (numbers indicate presence of regular, ringcontaining GDGTs) crenarchaeol and its regioisomer (Cren/Cren') and menaquinone-6 $\left(\mathrm{MK}_{6: 1}, \mathrm{MK}_{6: 0}\right)$ biosynthesis among cultivated members of the Archaea. Detailed distribution of GDGTs and GTGTs in Thaumarchaeota are shown in Table S1.

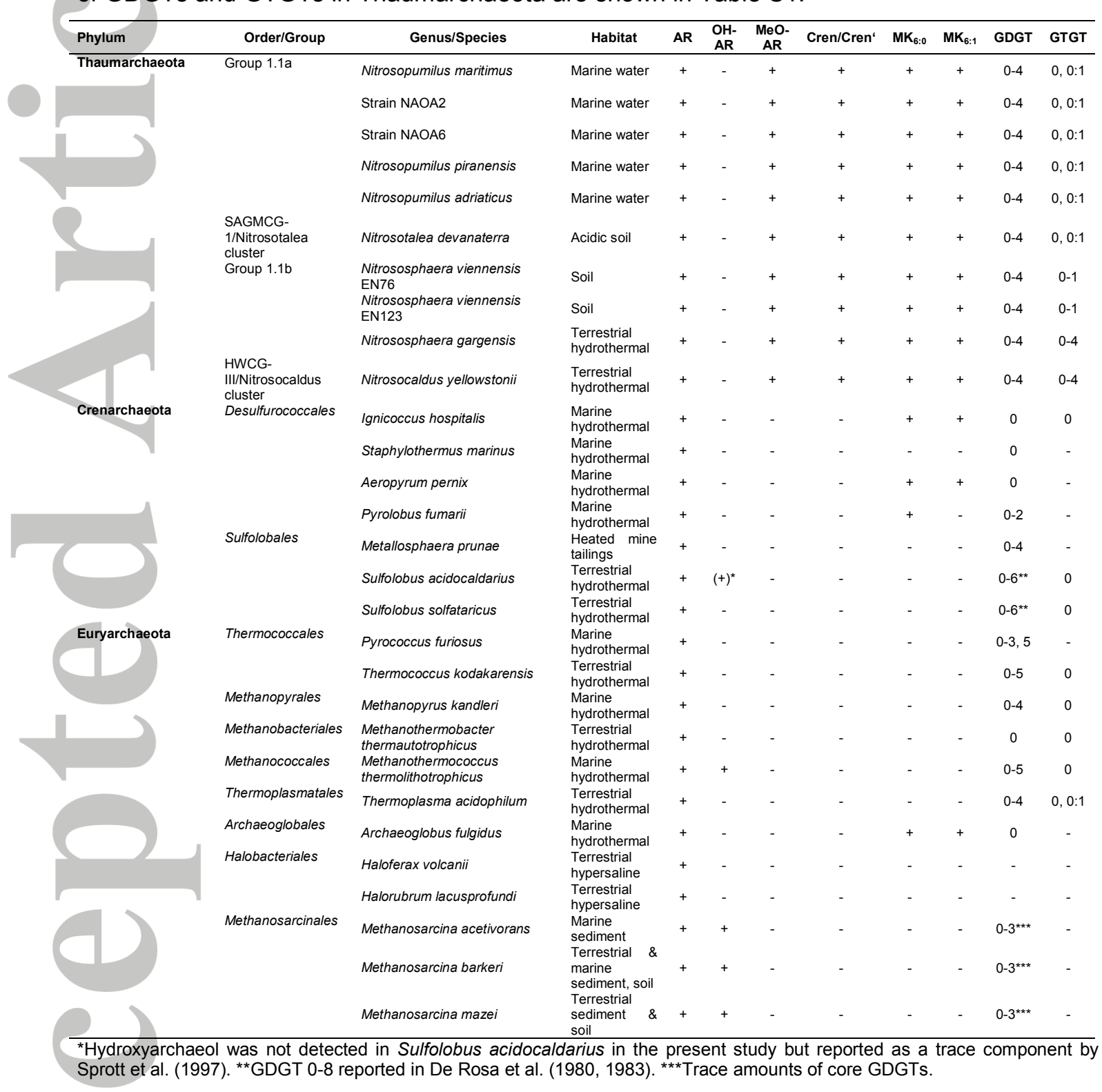

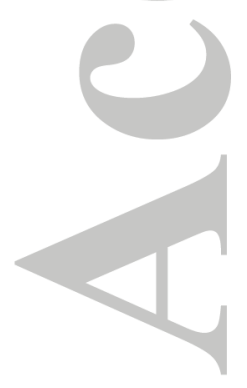

This article is protected by copyright. All rights reserved. 

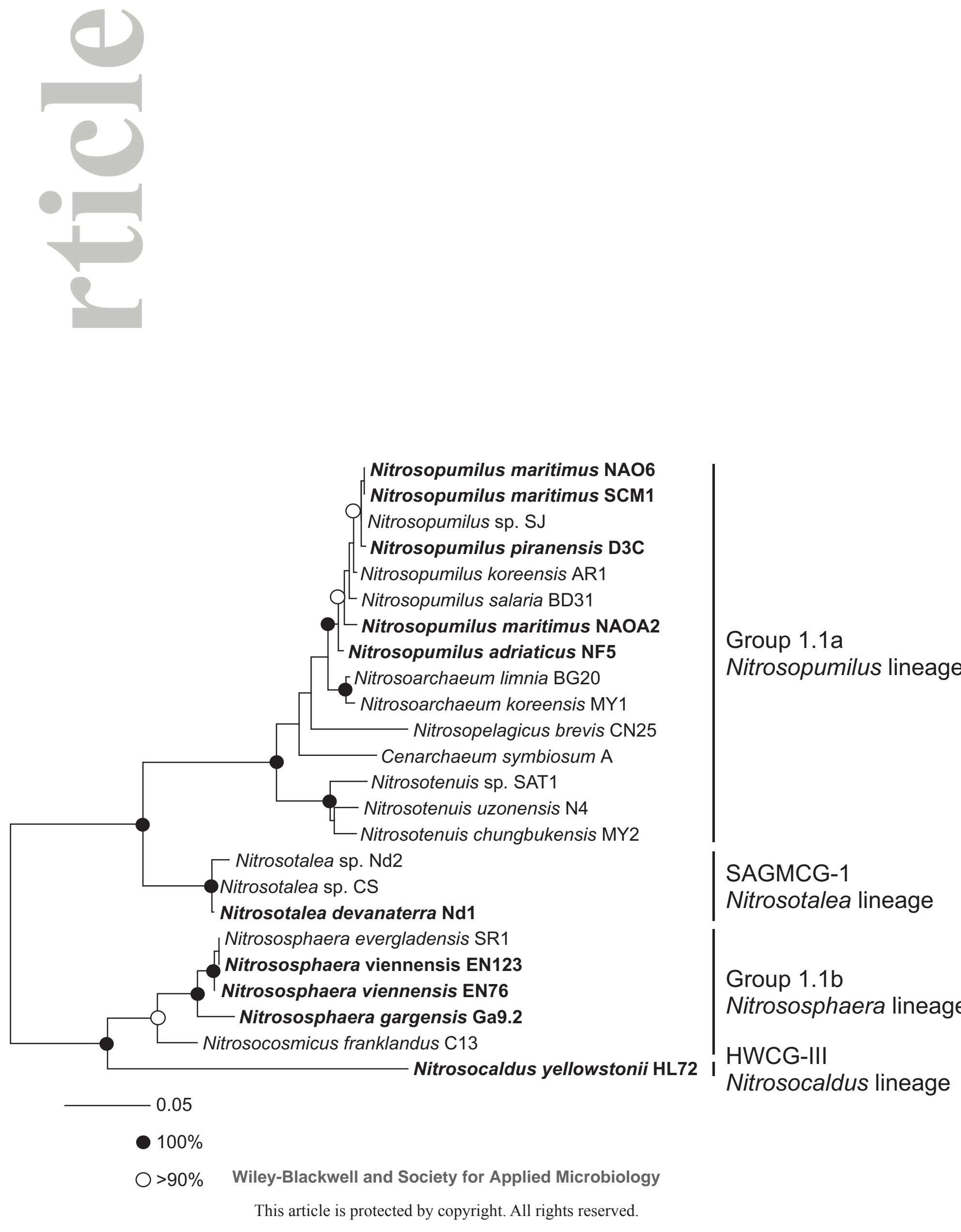

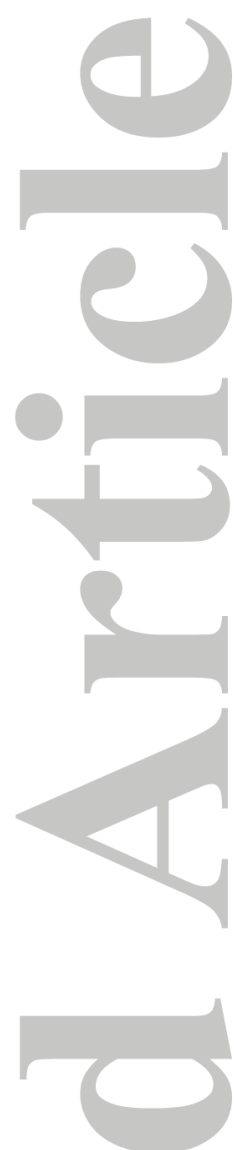

27 lipids detected exclusively in Group 1.1a: early 2G-GDGTs: $3-5$

2G-OH-GDGTs: $0,1,4$

early 2G-OH-GDGTs: $0-2$

Core OH-GDGTs: $0-4$

PH-GDGTs: $0-4$

1G-OH-GDGTs: $3-5$

1deoxyG-GDGTs: $0-3$

1G-unsGDGTs: 0 -2

2G-unsGDGTs:

9 lipids common to Groups $1.1 \mathrm{a}$ and $1.1 \mathrm{~b}$ : Archaeols: monouns.-AR, diuns.-AR PH-GDGTs: cren

HPH-GDGTs: 3 , cren'

1deoxyG-GDGTs: 1, 2, 4, cren

11 lipids detected exclusively in Group 1.1b: Archaeols: monouns.-MeO-AR, diuns.-MeO-AR 1deoxyG-GDGTs:cren'

1MeG-1G-GDGTs:4, cren, $X$

3G-GDGTs:4, cren

1MeG-2G-GDGTs:4, cren, cren'

\section{0 lipids common to}

Groups 1.1a, 1.1b, SAGMCG-1, and HWCG-III:

Archaeols:C-AR, MeO-AR, 1G-AR

Quinones: $\mathrm{MK}_{6 \cdot 0}, \mathrm{MK}_{6: 1}$

Core GDGTs: $0-5$, cren, cren'

MeO-GDGTs: 0-3, cren

Core GDDs: $1-4$, cren

1G-GDDs: $0-4$, cren

1G-GDGTs: $0-5$, cren, cren

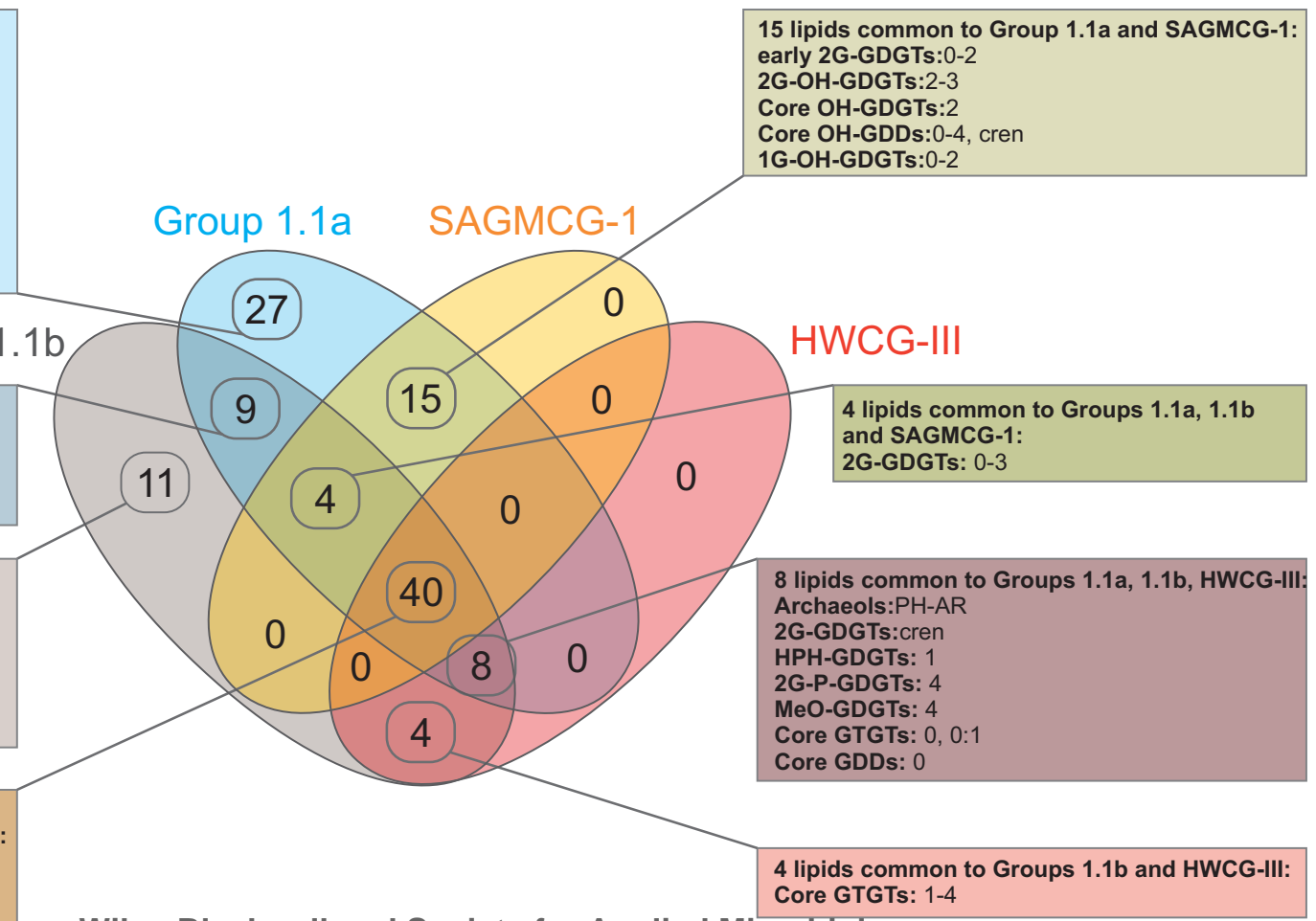

Wiley-Blackwell and Society for Applied Microbiology

This article is protected by copyright. All rights reserved. 


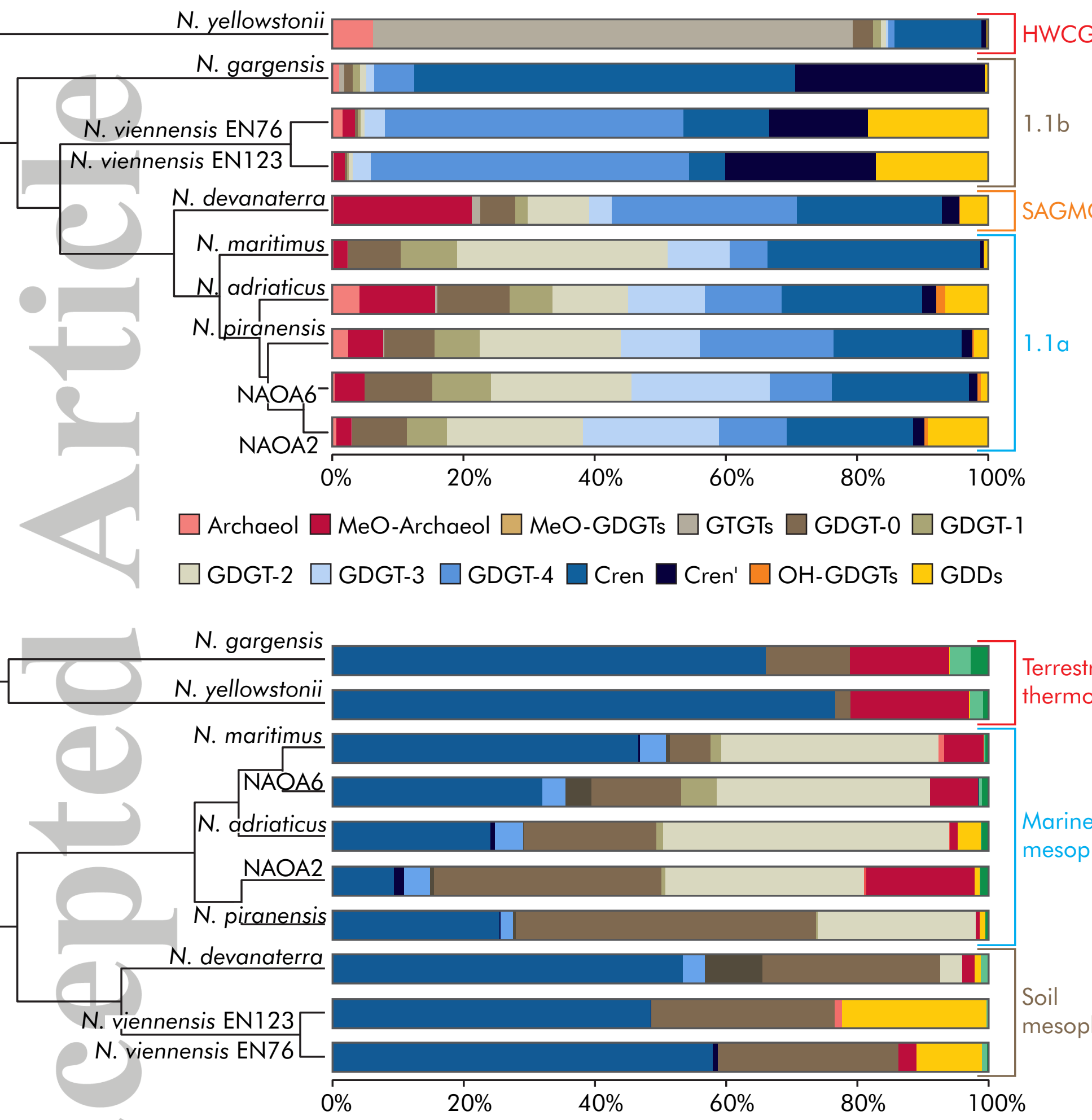

1G-GDGTs $\square$ deoxyG-GDGTs $\square$ 1G-OH-GDGTs $\square$ early2G-GDGTs $\square$ 2G-GDGTs $\square$ early2G-OH-GDGTs $\square$ 2G-OH-GDGTs $\square$ PH-GDGTs $\square$ HPH-GDGTs $\square$ 1G-GDDs $\square$ 1G-AR $\square$ PH-AR 

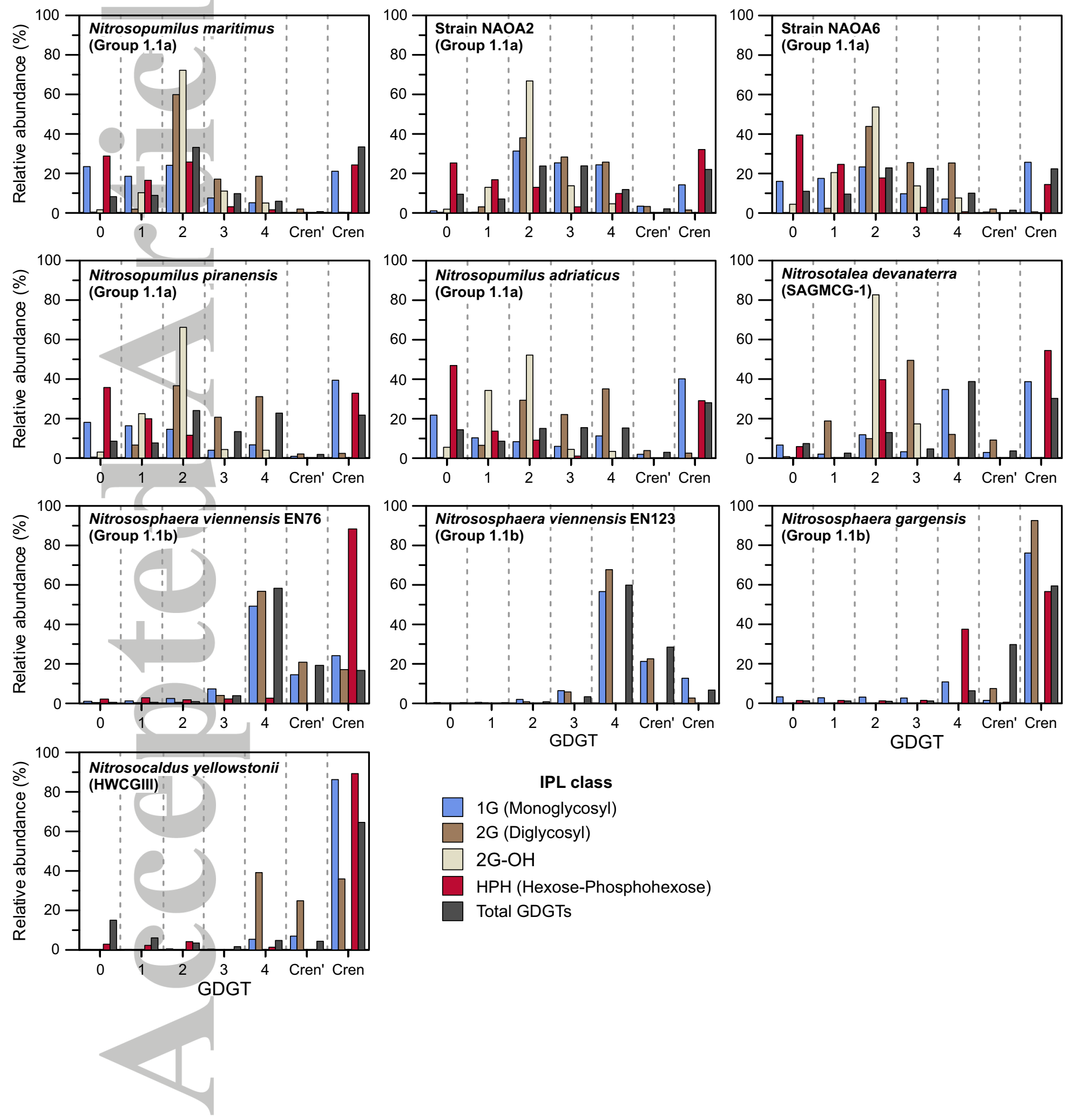

IPL class

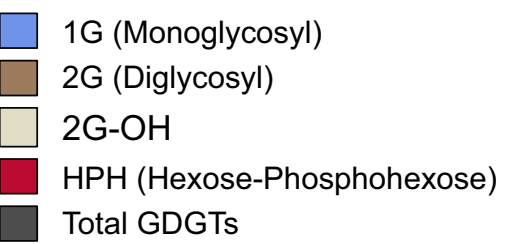


A
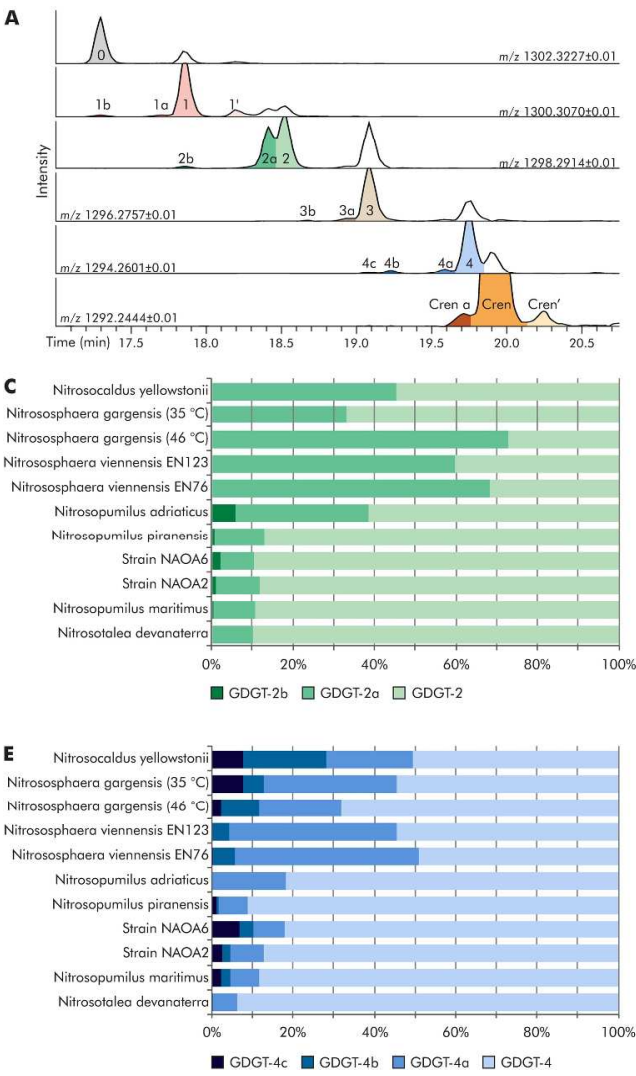

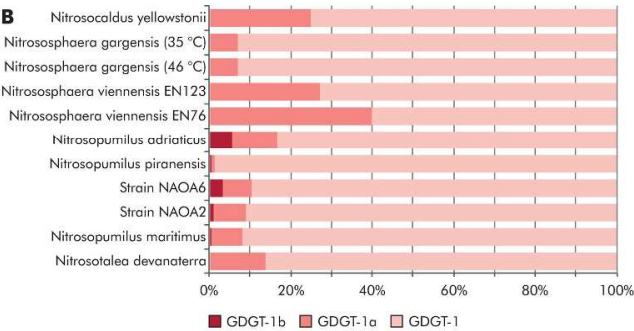

$\square$ GDGT-16 $\square$ GDGT-1a $\square$ GDGT-1
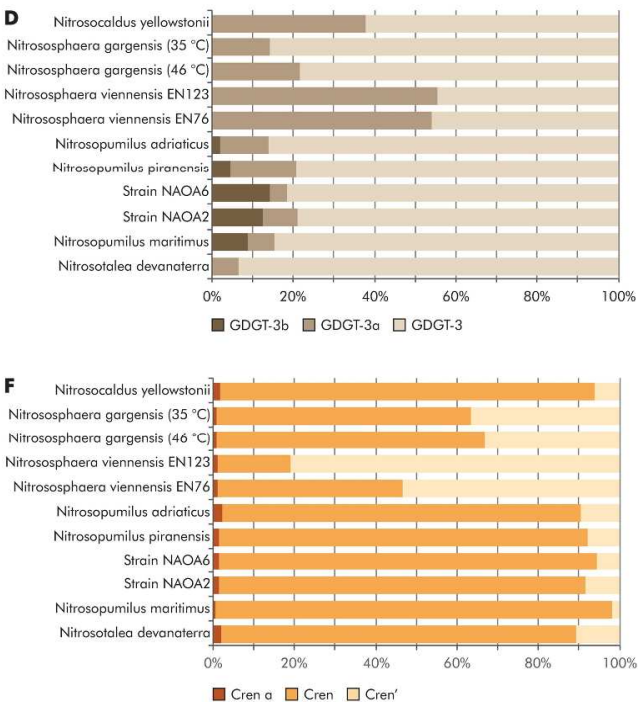

Fig. 6. (A) Extracted ion chromatograms showing elution of GDGT-1, $-2,-3,-4$, crenarchaeol and their isomers (a, b, c, cren') in a UPLC-APCI-MS analysis of a Ca. N. maritimus total lipid extract harvested in early growth phase (not used for panels B-F, intensity not to scale). Uncolored peaks in each chromatogram represent $+2 \mathrm{Da}$ isotope peaks of the respective lighter GDGT. (B to F) Relative abundances of GDGT-1, -2 , $-3,-4$, and crenarchaeol and their isomers in thaumarchaeal hydrolyzed total lipid extracts as determined using UPLC-APCI-MS (means of duplicate cultures).

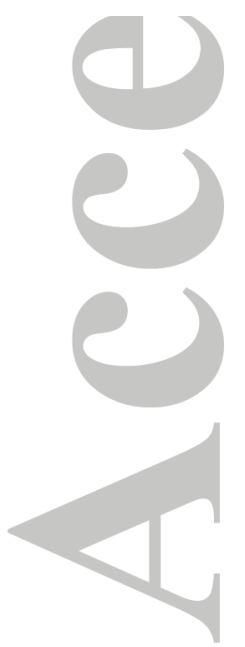

$243 \times 198 \mathrm{~mm}(300 \times 300 \mathrm{DPI})$

This article is protected by copyright. All rights reserved. 